\title{
Inhibitory control in neuronal networks relies on the extracellular matrix integrity
}

\author{
Egor Dzyubenko ${ }^{1}$ (D) Michael Fleischer ${ }^{1} \cdot$ Daniel Manrique-Castano $^{1} \cdot$ Mina Borbor $^{1} \cdot$ Christoph Kleinschnitz $^{1}$. \\ Andreas Faissner ${ }^{2}$. Dirk M. Hermann ${ }^{1}$
}

Received: 8 December 2020 / Revised: 11 May 2021 / Accepted: 19 May 2021 / Published online: 15 June 2021

(c) The Author(s) 2021

\begin{abstract}
Inhibitory control is essential for the regulation of neuronal network activity, where excitatory and inhibitory synapses can act synergistically, reciprocally, and antagonistically. Sustained excitation-inhibition (E-I) balance, therefore, relies on the orchestrated adjustment of excitatory and inhibitory synaptic strength. While growing evidence indicates that the brain's extracellular matrix (ECM) is a crucial regulator of excitatory synapse plasticity, it remains unclear whether and how the ECM contributes to inhibitory control in neuronal networks. Here we studied the simultaneous changes in excitatory and inhibitory connectivity after ECM depletion. We demonstrate that the ECM supports the maintenance of E-I balance by retaining inhibitory connectivity. Quantification of synapses and super-resolution microscopy showed that depletion of the ECM in mature neuronal networks preferentially decreases the density of inhibitory synapses and the size of individual inhibitory postsynaptic scaffolds. The reduction of inhibitory synapse density is partially compensated by the homeostatically increasing synaptic strength via the reduction of presynaptic $\mathrm{GABA}_{\mathrm{B}}$ receptors, as indicated by patch-clamp measurements and $\mathrm{GABA}_{\mathrm{B}}$ receptor expression quantifications. However, both spiking and bursting activity in neuronal networks is increased after ECM depletion, as indicated by multi-electrode recordings. With computational modelling, we determined that ECM depletion reduces the inhibitory connectivity to an extent that the inhibitory synapse scaling does not fully compensate for the reduced inhibitory synapse density. Our results indicate that the brain's ECM preserves the balanced state of neuronal networks by supporting inhibitory control via inhibitory synapse stabilization, which expands the current understanding of brain activity regulation.
\end{abstract}

Egor Dzyubenko

egor.dzyubenko@uk-essen.de

$\triangle$ Dirk M. Hermann

dirk.hermann@uk-essen.de

1 Department of Neurology and Center for Translational Neuro- and Behavioral Sciences (C-TNBS), University Hospital Essen, Hufelandstraße 55, 45122 Essen, Germany

2 Department of Cell Morphology and Molecular Neurobiology, Faculty of Biology and Biotechnology, Ruhr University Bochum, Universitätsstraße 150, 44801 Bochum, Germany 


\section{Graphic abstract}

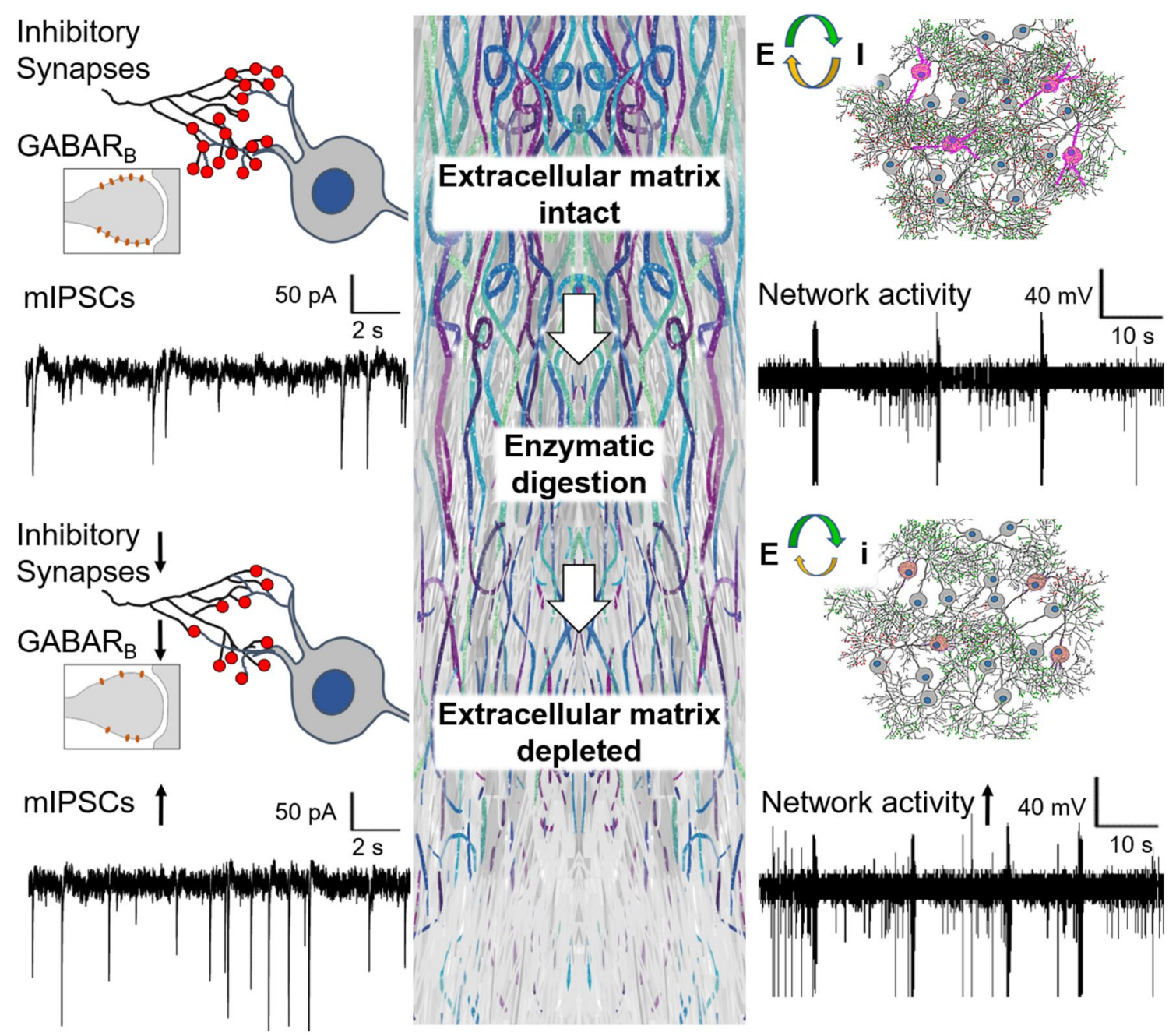

Keywords Neuronal network activity $\cdot$ ECM $\cdot$ Inhibitory synapse $\cdot$ E-I balance $\cdot$ Electrophysiology

\section{Introduction}

Neuronal activity is regulated through a dynamic balance of excitation and inhibition (E-I balance) [35] that requires coordinated plasticity of excitatory and inhibitory synapses $[6,69]$. In the brain, excitatory and inhibitory synapses are regulated cooperatively $[25,60]$, and their modulation can be synergistic, reciprocal, and antagonistic. Over several years, experimental studies have gathered solid evidence on the plasticity of individual excitatory synapses, establishing the modulation of presynaptic neurotransmitter release and postsynaptic responsiveness to glutamate as key neural correlates for memory and learning [39]. The co-regulation of excitatory and inhibitory synapses is less studied. On the network level, scaling of inhibitory synapses is essential for homeostatic mechanisms maximizing information processing capacity in neuronal networks [45]. Computational modelling suggests that patterns of neuronal network activity are primarily determined by inhibitory connectivity [47]. Yet, while the understanding of inhibitory synapse regulation continuously evolves $[13,29]$, our knowledge about how inhibitory plasticity modulates neuronal network activity at the integrative level, remains limited.

The brain extracellular matrix (ECM) is a multicomponent macromolecular meshwork containing chondroitin sulfate carrying proteoglycans (CSPGs), which anchors to the neuronal surface via hyaluronic acid synthesizing enzymes $[43,59]$. Neuronal activity induces the consolidation of ECM molecules [19] forming densely packed lattice-shaped layers around a subpopulation of neurons. These coatings, termed perineuronal nets (PNNs), support the fast-spiking properties of interneurons [16], regulate learning, and help 
to retain acquired memory [10]. In adulthood, CSPGs of PNNs and interstitial ECM restrict neuronal plasticity [11, 12, 20, 54]. A previous study [32] demonstrated that the lack of four major ECM proteoglycans (tenascin-C, tenascin-R, brevican, and neurocan) compromises PNN integrity and alters neuronal network development. In the present work, we explored how ECM depletion affects mature neuronal networks, in which earlier developmental stages were not augmented via genetic manipulation.

Enzymatic digestion of the brain ECM increases lateral mobility of excitatory glutamate receptors, alters short-term plasticity of excitatory synapses [28] and enhances neuronal network activity [7]. Thus, ECM integrity is essential for sustained synaptic signaling and neuronal circuit stability. Upon brain injury induced by ischemic stroke, the ECM is rapidly degraded within a few hours [36], and it stays partly decomposed after one week in lesion-surrounding brain areas [22], in which neuronal network activity is compromised $[17,44]$. To understand how ECM alterations affect neuronal activity after brain injury, it is necessary to investigate the adjustment of neuronal networks at the integrative level after ECM depletion by bridging synaptic, neuronal, and network activity changes. Despite the link between ECM integrity and inhibitory interneuron function was previously demonstrated [4, 5, 67], the role of inhibitory synapse plasticity in controlling network activity after ECM depletion remained unknown.

Here we investigated the role of the brain ECM for stabilizing excitatory and inhibitory synapses and balancing neuronal network activity. We enzymatically degraded hyaluronic acid and CSPGs in the extracellular space and combined structural and functional readouts for studying neuronal connectivity and activity at the synapse and network level. By using data-driven computer simulations, we explored the influence of synaptic strength and connectivity changes on the activity of neuronal networks.

\section{Materials and methods}

\section{Legal issues and animal housing}

Experimental procedures were approved by the local government (Bezirksregierung Düsseldorf) and conducted in accordance with European Union (Directive 2010/63/ EU) guidelines for the care and use of laboratory animals. C57BL/6j mice (Envigo, Indianapolis, IN, U.S.A.) were kept in groups of 5 animals/cage in a regular inverse $12 \mathrm{~h}$ light-dark cycle and access to food and water ad libitum. All efforts were made to reduce the number of animals in the experiments.

\section{Cell cultures}

Primary cultures of neurons and astrocytes were prepared as described previously $[31,33]$. Hippocampal neurons were obtained at embryonic day 15 (E15) and cortical astrocytes were obtained at postnatal day 1 (P1) from male and female mice. Neurons were supported by astrocyte monolayers cultivated on cell culture inserts with a permeable membrane (Fig. S1A), allowing for the long-term maturation of neuronal networks. We plated 50,000 neurons in a $50 \mu \mathrm{l}$ droplet onto pre-treated glass coverslips or MEA chips. Inserts containing 50,000 astrocytes were combined with neuronal cultures on the same day. The cultures were maintained in Neurobasal medium (21103049, ThermoFisher, Waltham, MA, U.S.A.) supplemented with $2 \mathrm{mM} \mathrm{L-glutamine} \mathrm{(25030081,}$ ThermoFisher), 1\% v/v B27 (A3582801, ThermoFisher) and 1\% v/v SM1 (05711, STEMCELL Technologies, Vancouver, Canada). We changed half of the medium weekly to keep the $\mathrm{pH}$ around 6.5. All in vitro experiments were performed on fully mature neurons after 21-28 days of cultivation (Figs. $\mathrm{S} 1$ and S2). The mature networks consisted of $\sim 67 \%$ principal excitatory neurons and $\sim 33 \%$ inhibitory interneurons, which is slightly higher than $20-25 \%$ in vivo $[38,66]$. Virtually all inhibitory cells were parvalbumin-containing, and the percentage of other interneuron types could be therefore neglected [21]. The evolution of neuronal networks in vitro was characterized by coherent maturation of synaptic connectivity and ECM expression, partially resembling neuronal circuit development in vivo [14]. Synaptic connectivity of neuronal networks in vitro established randomly, mimicking the organization of cortical networks [3, 61]. During cultivation, synapse density increased until maturity was reached after 21 days in vitro (DIV) (Fig. S1B, C). The establishment of network connectivity correlated with the expression of PNNs, the condensed ECM layers characteristic for the mature neuronal cultures (Fig. S1B, D). Network activity in neuronal cultures evolved accordingly, changing from the high-frequency random spiking at 7 DIV followed by the low activity period at 14 DIV and the regular bursting pattern at 21 DIV (Fig. S1E). At later time points (i.e., 35 DIV) synapse density, PNN number, and network activity were stabilized and did not change. The mature neuronal cultures contained $34 \pm 6.3 \%$ (mean \pm s.e.m.) inhibitory interneurons (Fig. S2).

\section{ECM depletion}

We followed an established approach for enzymatic ECM digestion $[7,54,56]$. The treatment solutions were labelled in a non-descriptive manner (i.e. A, B, C), making the experimenter blinded during the treatment, data acquisition, and data analysis. Cell cultures were incubated with $500 \mathrm{mU} / \mathrm{ml}$ ChABC (C3667, Sigma-Aldrich, Taufkirchen, Germany) or 
$500 \mathrm{U} / \mathrm{ml}$ hyaluronidase (HYase, H4272, Sigma-Aldrich) for $16 \mathrm{~h}$. For ECM depletion in vivo, $500 \mathrm{mU}$ ChABC or 500 $\mathrm{U}$ HYase were dissolved in $2 \mu \mathrm{l}$ of $0.1 \mathrm{M}$ PBS and delivered via a single stereotactic (bregma 0 , left $3 \mathrm{~mm}$, deep $1 \mathrm{~mm}$ ) intracortical injection. At $16 \mathrm{~h}$ post-injection, animals were sacrificed and brains were processed for further analysis. Control animals were treated with a vehicle (0.1 M PBS). In our hands, the two enzymes were equally efficient for ECM digestion and specifically targeted the neuronal cell culture compartment, while no alterations in glial cells were detected (Fig. S3). Moreover, ChABC and HYase induced identical changes in synapse density and network activity (Fig. S4).

\section{Immunolabelling procedures}

For immunohistochemistry, brains were perfused with $4 \%$ $\mathrm{w} / \mathrm{v}$ paraformaldehyde (PFA) and post-fixed for $12 \mathrm{~h}$ in $4 \%$ w/v PFA. $40 \mu \mathrm{m}$ coronal free-floating sections were obtained from the bregma level. For immunocytochemistry, cell cultures were fixed with $4 \%$ w/v PFA for $10 \mathrm{~min}$ at room temperature. Synaptic proteins were detected mouse anti-PSD95 (1:500, MAB1598, Millipore, Burlington, MA, U.S.A.), guinea pig anti-VGLUT1 (1:500, 135304, Synaptic Systems, Goettingen, Germany), mouse anti-gephyrin (1:500, 147011, Synaptic Systems) and guinea pig anti-VGAT $(1: 500,131103$, Synaptic Systems) antibodies. For GABA receptor quantification, $\mathrm{GABA}_{\mathrm{A}}$ and $\mathrm{GABA}_{\mathrm{B}}$ receptors were labelled with chicken anti-GABA ${ }_{A} \gamma 2(1: 500,224006$, Synaptic Systems) and rabbit anti-GABA B $(1: 500,322102$, Synaptic Systems) antibodies. To characterize the ECM expression, we applied biotinylated WFA (1:100, B-1355, Vector Laboratories, Burlingame, USA), biotinylated hyaluronan binding protein (1:100, 400763, AMS Biotechnology, Frankfurt, Germany), rabbit anti-aggrecan antibody (1:500, AB1031, Millipore) and rat anti-473HD antibody (1:100, produced by the group of Prof. Andreas Faissner, Bochum, Germany; [72]). Neuronal types were identified using rabbit anti-GABA (1:2000, A2052, Sigma-Aldrich, chicken anti-NeuN (1:300, ABN91, Millipore, mouse antineurofilament M (1:500, 171231, Synaptic Systems and rabbit anti-Kv3.1b (1:1000, APC-014, Alomone Labs, Jerusalem, Israel antibodies. Rat anti-glial acidic fibrillary protein (GFAP; 1:1000, 13-0300, ThermoFisher, mouse anti- $\beta$ catenin (1:500, ab19381, Abcam, Cambridge, UK and rabbit anti-connexin 43 (1:500, 3512, Cell Signaling Technologies, Frankfurt, Germany antibodies were used as astroglial markers. For fluorescence detection, secondary antibodies conjugated to Alexa or Atto dyes were used. Nuclei were counterlabeled with DAPI (1:1000, D1306, ThermoFisher).

\section{Low-resolution microscopy for basic quantifications}

For basic quantifications of cell density and marker proteins expression following immunohistochemistry/ immunocytochemistry, four $425.1 \times 425.1 \mu \mathrm{m}$ regions of interest (ROIs) per condition per experiment were selected at random positions for cell culture specimens. In brain sections, the ROIs were positioned in the left and right cerebral somatosensory cortex layers $3-5$. Single plane micrographs were obtained using the Carl Zeiss LSM710 confocal microscope (20×Plan Apochromat objective, NA 0.8).

\section{Total expression of GABA receptors}

The total expression of GABA receptors on the neuronal surface was investigated in vitro by Western blot. Membrane proteins were extracted using the Mem-PER Plus Membrane Protein Extraction Kit (89842, ThermoFisher) and separated by sodium dodecyl sulfate polyacrylamide gel electrophoresis (SDS-PAGE) on $1 \mathrm{~mm} \mathrm{8 \%} \mathrm{polyacrylamide} \mathrm{gels.} \mathrm{To} \mathrm{avoid}$ protein aggregation the samples were not heated. The proteins were transferred onto nitrocellulose membranes using the Trans-Blot Turbo Transfer System (Biorad, Hercules, CA, U.S.A.) mixed molecular weight program, followed by pre-blocking in $3 \%$ bovine serum albumin (BSA) for $1 \mathrm{~h}$. The membranes were incubated with primary chicken anti-GABA $_{A} \gamma 2$ (1:1000, 224006, Synaptic Systems), rabbit anti-GABA $A_{B}(1: 1000,322102$, Synaptic Systems) and mouse anti-synaptotagmin-1 (1:1000, 105011, Synaptic Systems) antibodies for $72 \mathrm{~h}$ at $4{ }^{\circ} \mathrm{C}$. Secondary antibodies were applied stepwise, and the proteins were visualized in separated fluorescence and luminescence channels. For fluorescence detection, Alexa-647 and Cy-3 conjugated antibodies were used. Chemiluminescence was detected with HRP conjugated antibodies using Pierce ECL Western blotting substrate (32106, ThermoFisher). Multiple proteins were detected on the same membrane using the ChemiDoc XRS + Imaging System (Biorad), and labelling intensity was normalized to the stain-free signal. The molecular weights of the proteins were verified using a prestained protein ladder (ab116028, Abcam). Data were analyzed by densitometry in ImageJ using the gel quantification plugin.

\section{Synapse density and synaptic GABA receptor quantifications}

The density of glutamatergic and GABAergic synapses was quantified using a previously established method [23]. For synapse analysis in vitro, five $66.5 \times 66.5 \times 5 \mu \mathrm{m}$ ROIs per condition per experiment were selected at random positions, containing the soma and proximal dendrites of single neurons. In brain sections, five $51 \times 51 \times 10 \mu \mathrm{m}$ ROIs per condition per experiment were selected in the left 
and right cerebral cortex layers 3-5. The confocal stacks were obtained using the LSM710 confocal microscope (100×alpha Plan-Apochromat objective, NA 1.46; Carl Zeiss, Jena, Germany). Structurally complete synapses were identified by the overlapping immunolabelling of pre- and postsynaptic markers and quantified with the Synapse Counter plugin for ImageJ (https://github.com/SynPuCo/Synap seCounter). Our synapse quantification approach detects the majority of inputs, which a particular cell receives from its local network partners. The expression of pre- and postsynaptic $\mathrm{GABA}_{\mathrm{B}}$ and $\mathrm{GABA}_{\mathrm{A}}$ receptors was evaluated by immunofluorescence intensity analysis. Pre- and postsynaptic structures were analyzed using Synapse Counter, and mean pixel intensities were determined as estimates of protein expression changes.

\section{STED microscopy of postsynaptic scaffolds}

The morphology of postsynaptic scaffolds was investigated by STED microscopy using a previously established method [23]. We employed the time-gated Leica TCS SP8 microscope (Wetzlar, Germany), which is equipped with a white light pulse laser (WLL2) and gated hybrid detection. An oil immersion HCX PL APO STED 100x (numerical aperture 1.4) objective was used. The ultrastructure of postsynaptic scaffolds within structurally complete synapses was analyzed. First, $23.8 \times 23.8 \mu \mathrm{m}$ single-plane confocal images were obtained using 488 and $633 \mathrm{~nm}$ excitation wavelengths for the post- and presynaptic markers, respectively. Then, the STED scans were obtained in the same stage position using $488 \mathrm{~nm}$ excitation and $592 \mathrm{~nm}$ depletion lasers. The detection time-gating interval was set to $6-10$ ps post-pulse time window. All settings were the same throughout the experiments. The raw data were deconvolved using Hyugen's software. The binary masks of single synaptic scaffolds were generated using automated thresholding (Otsu method) in ImageJ, and the mask area was measured.

\section{Whole-cell patch-clamp recordings}

Action potential generation and spontaneous postsynaptic currents were measured in mature neuronal cultures by the whole-cell patch-clamp method using Axopatch 200B amplifier (Molecular Devices, San Jose, CA, U.S.A.) and analyzed with pClamp software 10.6 (Molecular Devices). Microelectrodes of $1.5 \mathrm{~mm}$ thin-walled filamented borosilicate glass (World Precision Instruments, Friedberg, Germany) were pulled with a DMZ-Universal Puller (Zeitz Instruments, Martinsried, Germany) and polished to a final resistance of 3-4 M . Neurons were voltage-clamped at $-60 \mathrm{mV}$, the signals were filtered at $1.0 \mathrm{kHz}$ and recorded with $10 \mathrm{kHz}$. Series resistance and cell capacitance were compensated prior to the recordings. BrainPhys basal medium (05790,
STEMCELL Technologies) was used as an extracellular solution. The pipette solution contained $140 \mathrm{mM} \mathrm{KCl}$, $1 \mathrm{mM} \mathrm{CaCl} 2 \cdot 2 \mathrm{H}_{2} \mathrm{O}, 4 \mathrm{mM} \mathrm{MgCl}{ }_{2}, 10 \mathrm{mM}$ HEPES, $0.4 \mathrm{mM}$ $\mathrm{Na}_{2}$-GTP, $4 \mathrm{mM} \mathrm{Mg-ATP}$ and $10 \mathrm{mM}$ EGTA (pH 7.3). Action potential thresholds were determined by applying incrementally increasing depolarizing current steps of $500 \mathrm{~ms}$ duration. Passive membrane properties and firing patterns were characterized to discriminate the fast-spiking inhibitory interneurons (spiking frequency $\geq 5 \mathrm{~Hz}$ ) and the excitatory neurons (spiking frequency below $5 \mathrm{~Hz}$ ). For the recording miniature inhibitory postsynaptic currents (mIPSCs) we applied $1 \mu \mathrm{M}$ tetrodotoxin (TTX, Tocris, Bristol, UK) to prevent action potential-driven synaptic release. The inhibitory postsynaptic currents were pharmacologically isolated using $10 \mu \mathrm{M}$ glutamate receptor antagonist DNQX (Tocris) and $10 \mu \mathrm{M}$ NMDA receptor antagonist D-APV (Tocris).

\section{Spontaneous network activity recordings}

Spontaneous activity in neuronal networks was measured using cell culture compatible square $8 \times 8$ electrode MEA (60MEA200/30iR-Ti, Multi Channel Systems, Reutlingen, Germany), on which primary neurons were grown. To evaluate the effects of ECM depletion, we recorded the baseline activity prior to the application of digesting enzymes and compared it with the post-treatment activity after $16 \mathrm{~h}$. The control cultures were treated with 0.1 M PBS. To study the impact of ECM depletion on GABAergic neurotransmission, $\mathrm{GABA}_{\mathrm{A}}(6 \mu \mathrm{M}$ bicuculline metiodide, Tocris) or $\operatorname{GABA}_{B}(100 \mu \mathrm{M}$ CGP46381, Tocris) receptor antagonists were applied. After 30 min of incubation with the antagonist, network activity was recorded. The effects of GABA antagonists were evaluated with reference to neuronal activity after ECM depletion or control treatment. In all experiments, we recorded spontaneous network activity for $15 \mathrm{~min}$ (temperature stabilized at $35^{\circ} \mathrm{C}$, gas exchange prevented) using the MEA2100 60-channel headstage with the sampling frequency of 40,000 Hz using MC Rack. For each electrode, the mean firing rate (MFR) and mean bursting rate (MBR) were analyzed in MatLab with the SpyCode toolbox generously provided by Dr. Michela Chiappalone [9].

\section{Network activity simulations}

To evaluate the impact of connectivity alterations versus the synaptic strength changes induced by ECM depletion, we implemented a previously established computational approach [21]. Experimentally observed effects of ECM depletion were reconstructed using an in silico network of spiking neurons. The key variables of our model define neuronal response properties and network connectivity. The physiology of fast-spiking interneurons and primary 
excitatory neurons was replicated using previously defined parameters [41]. Network circuitry was defined by the sparseness of connectivity $\left(\mathrm{C}_{\mathrm{exc}}, \mathrm{C}_{\text {inh }}\right)$ and synaptic weights $\left(\mathrm{W}_{\mathrm{exc}}, \mathrm{W}_{\mathrm{inh}}\right)$. The sparseness of connectivity was defined as the proportion of all neurons of a certain type providing the input to a single cell on average: $C_{\mathrm{exc}}=0.25 * \mathrm{~N}_{\mathrm{exc}}$ for excitatory, $C_{\text {inh }}=0.15^{*} \mathrm{~N}_{\text {inh }}$ for inhibitory input in the control condition. Synaptic weights were set as absolute values of postsynaptic membrane potential changes after activation of a synapse. We modified $\mathrm{C}_{\text {inh }}$ and $\mathrm{W}_{\text {inh }}$ within a range of biologically feasible values to determine their impact on neuronal network activity. For each simulation instance, network connectivity was newly generated, mimicking the intrinsic variability of real neuronal networks.

\section{Statistics}

For non-normally-distributed datasets, data were evaluated by Kruskal-Wallis tests using OriginPro2020 software. For multiple comparisons, Bonferroni correction was applied. Data were presented as box plots depicting the medians (lines inside boxes)/ means (filled squares inside boxes) \pm IQR (boxes) with $10 \%$ and $90 \%$ ranks as whiskers. For normally distributed datasets, e.g. the in silico simulations, data were evaluated by two-tailed independent Student's t-tests. Data were shown as mean \pm s.e.m. columns. Data points were indicated as diamonds. With the significance level $\alpha$ set to 0.05 , the $p$ values $<0.05$ were defined to indicate statistically significant differences.

\section{Results}

\section{ECM depletion reduces inhibitory synapse density in vitro}

Measuring synapse density changes provides an indirect but straightforward estimate of neuronal network connectivity alterations [21]. Thus, we first quantified the density of structurally complete synapses in mature networks of primary murine neurons. The networks consisted of $\sim 67 \%$ principal excitatory neurons and $33 \%$ inhibitory interneurons (which is slightly higher than $20-25 \%$ in vivo $[38,66]$ ) and were fully mature after 21 days of cultivation, indicated by the appearance of PNNs, which are condensed ECM layers, in neuronal cultures (Figs. S1 and S2). The density of both GABAergic and glutamatergic synapses was quantified using GABA as a marker of inhibitory perikarya, allowing for the gross estimation of network connectivity. The co-labelling of vesicular glutamate transporter type 1 (VGLUT1) and postsynaptic density protein 95 (PSD95) indicated the excitatory inputs to excitatory and inhibitory neurons, while the co-labelling of vesicular GABA transporter (VGAT) and gephyrin signified inhibitory inputs to excitatory and inhibitory neurons (Fig. 1a, b).

The density of glutamatergic and GABAergic synapses was differently affected by enzymatic ECM depletion (500 mU/ml chondroitinase ABC [ChABC], 16 h). Compared with the control, ECM depletion reduced excitatory input to inhibitory neurons by $11.2 \pm 5.5 \%$, inhibitory input to excitatory neurons by $60.3 \pm 5.7 \%$ and inhibitory input to inhibitory neurons by $53.7 \pm 5.9 \%$ (Fig. 1c). ECM depletion did not affect neuronal survival (Fig. S5). Because GABAergic synapses were affected more strongly than glutamatergic ones, we concluded that ECM depletion preferentially reduced inhibitory connectivity in vitro.

\section{ECM depletion reduces inhibitory synapse density in vivo}

Further, we analyzed the density of glutamatergic and GABAergic synapses in somatosensory cortex layers 3-5 following ECM depletion in vivo. Intracortical injection of ChABC (500 mU in $3 \mu 10.1 \mathrm{M}$ phosphate-buffered saline [PBS], $16 \mathrm{~h}$ ) unilaterally depleted ECM in the brain, indicated by the absence of PNNs, which in the brain are found around the fast-spiking interneurons expressing a specific potassium channel Kv3.1. The procedure did not affect the density of $\mathrm{Kv} 3.1^{+}$neurons and did not alter PNN expression in the contralateral hemisphere (Fig. 2a, c). ECM depletion did not influence the density of glutamatergic synapses but significantly reduced GABAergic synapses in the cortex by $42 \pm 6 \%$ (Fig. 2b, d). Hence, ECM depletion reduced inhibitory connectivity in vivo.

\section{ECM depletion alters electrophysiological properties of neurons}

To investigate whether the reduced inhibitory connectivity after ECM depletion is accompanied by neuronal electrophysiology changes, we analyzed passive membrane properties (resting membrane potential and capacitance) and action potential generation (threshold, frequency) using somatic patch-clamp recordings (Fig. 3a, b). We applied incrementally increasing depolarizing current steps of $500 \mathrm{~ms}$ duration to determine action potential thresholds in excitatory and inhibitory neurons. Neuronal types were discriminated against by their spiking behavior and resting membrane potentials. Like in previous studies [46, 57, 68], the inhibitory interneurons exhibited considerably faster spiking dynamics (spiking frequency $\geq 5 \mathrm{~Hz}$ ) than the excitatory neurons (spiking frequency below $5 \mathrm{~Hz}$ ). ECM depletion significantly increased the spiking frequency of inhibitory interneurons but did not alter action potential threshold, resting membrane potential, and capacitance (Fig. 3a). In excitatory neurons, ECM depletion reduced the action potential 

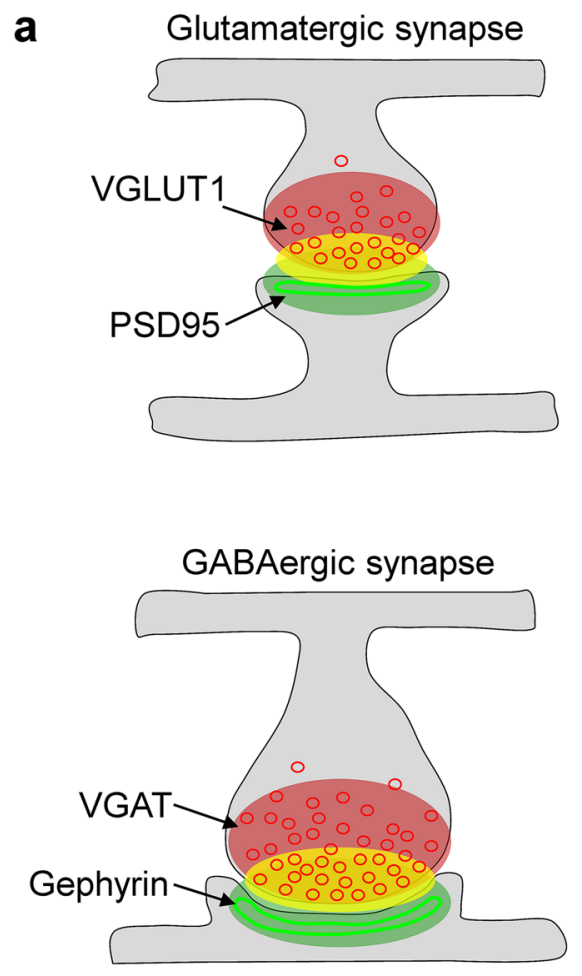
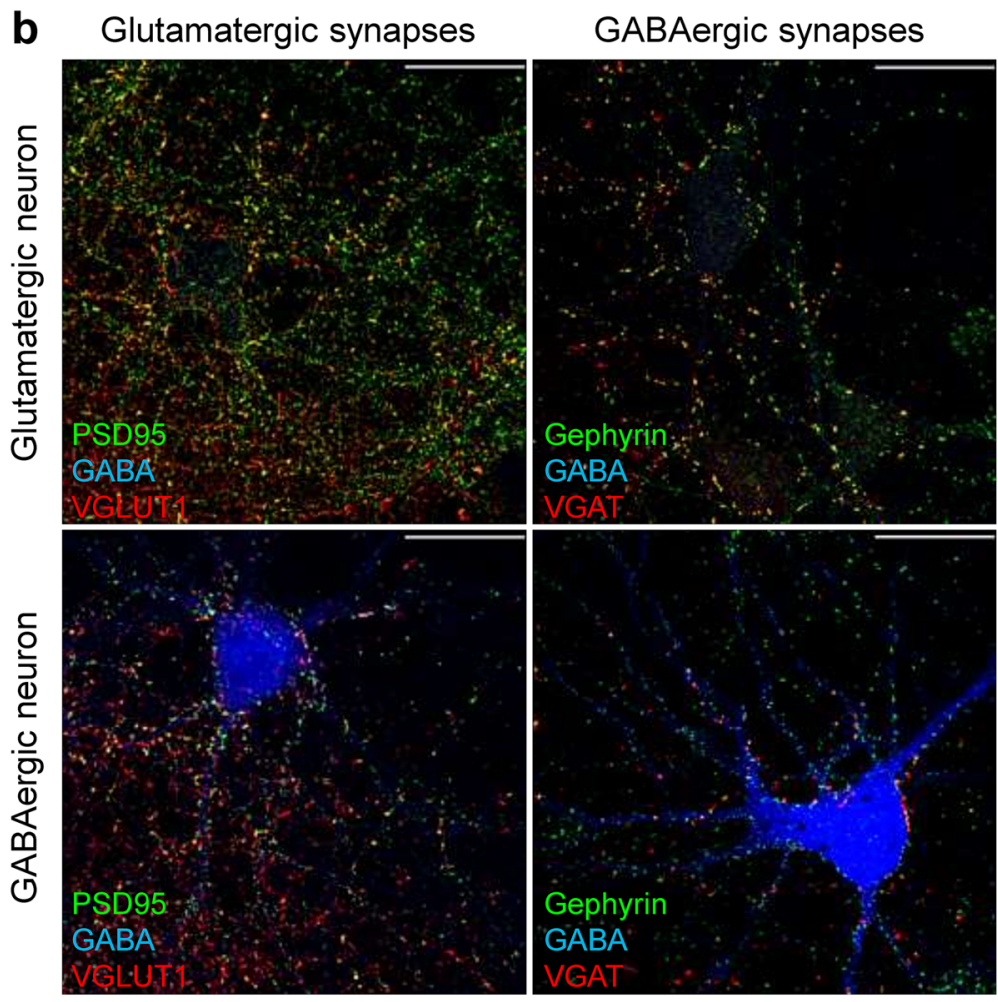

\begin{abstract}
Excitatory input to inhibitory neurons
\end{abstract}

Inhibitory input to excitatory neurons
Inhibitory input to inhibitory neurons
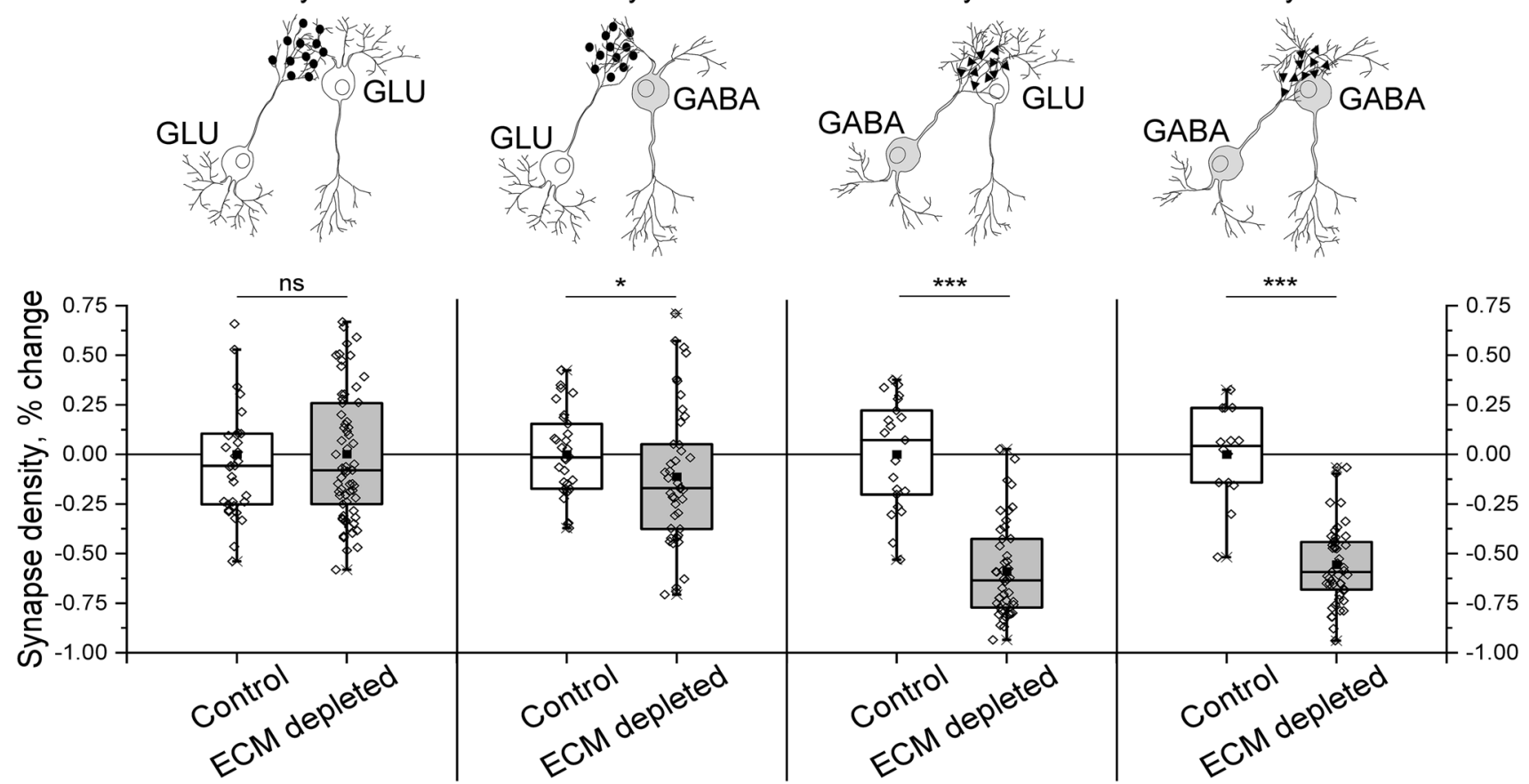

Fig. 1 Excitatory and inhibitory synapse densities decrease after ECM depletion in vitro. a Overlapping immunolabelling of presynaptic (red) and postsynaptic (green) markers was used to detect structurally complete synapses (yellow). b The density of glutamatergic (PSD95-VGLUT1) and GABAergic (gephyrin-VGAT) synapses was measured with reference to GABA immunoreactivity. Representative micrographs are shown. Scale bars, $30 \mu \mathrm{m}$. c Synapse density changes were calculated as differences with mean values of corre- sponding control experiments. Data are shown for each neuron examined ( $n \geq 20$ neurons per condition, results obtained from 5 independent experiments). GLU glutamate. Data are medians (lines inside boxes)/ means (filled squares inside boxes) \pm IQR (boxes) with 10/ $90 \%$ ranks as whiskers. Open diamonds are data points. The asterisks indicate significant differences with control, based on Kruskal-Wallis tests $(* * * p<0.001, * * p<0.01)$. $n s$ not significant 

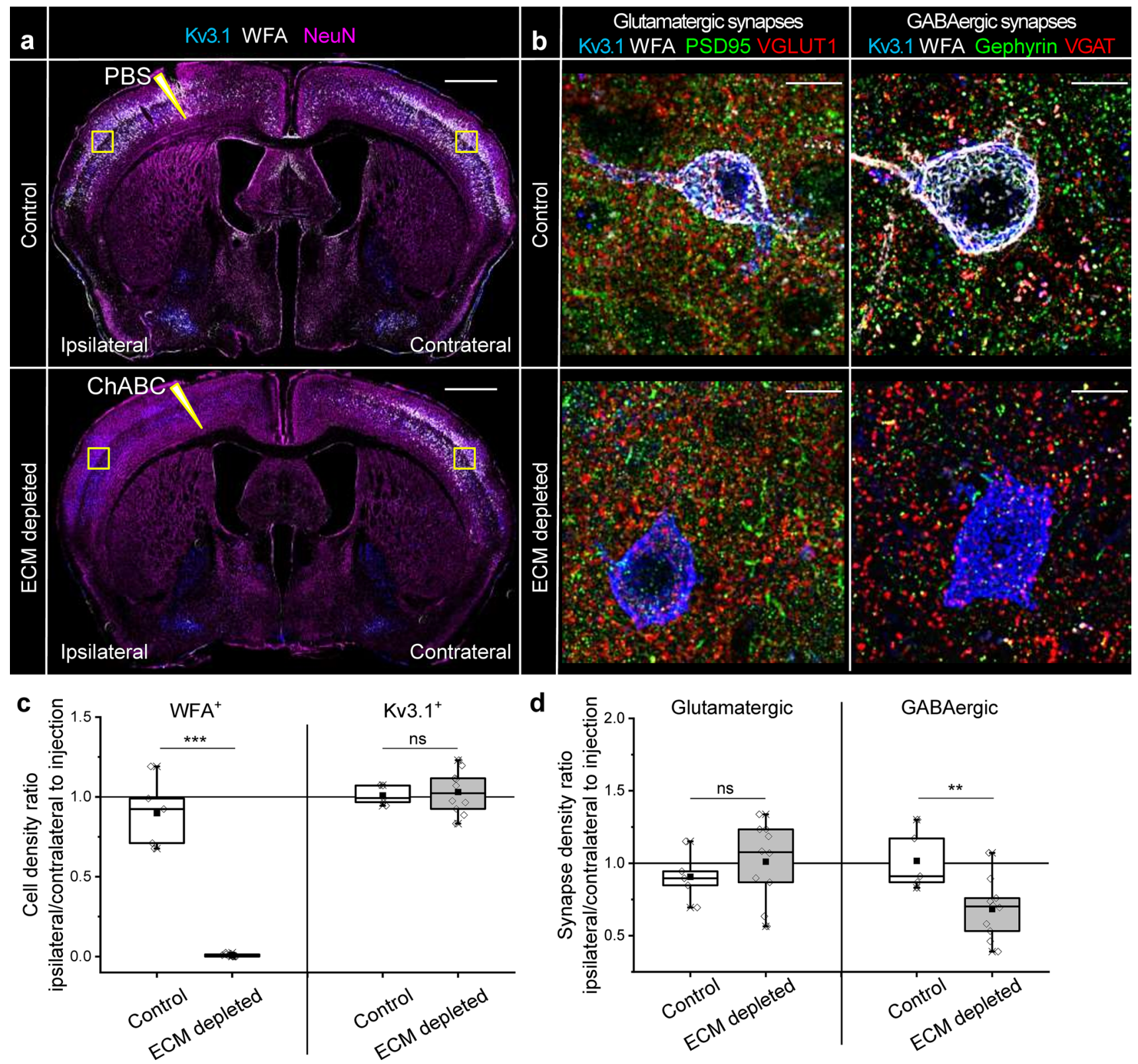

Fig. 2 Inhibitory synapse density decreases after ECM depletion in vivo. a Neuronal nuclei (NeuN, magenta), fast spiking interneurons (Kv3.1, blue) and PNNs (WFA, Wisteria floribunda agglutinin, white) were immunohistochemically labeled in brain sections obtained from mice treated with chondroitinase $\mathrm{ABC}$ (ChABC, ECM depleted) or phosphate buffered saline (PBS, control) for $16 \mathrm{~h}$. Sharp triangles indicate intracortical injection sites. Squares indicate the regions in which cell and synapse densities were analyzed. Scale bar, $1 \mathrm{~mm}$. b The density of glutamatergic (PSD95-VGLUT1) and GABAergic (gephyrin-VGAT) synapses was measured in somatosensory cortex layers $3-5$. Maximum projections of $56.7 \times 56.7 \times 5$

$\mu \mathrm{m}$ regions ipsilateral to the injection sites are shown. Scale bars, $10 \mu \mathrm{m}$. $\mathbf{c}$ Changes in $\mathrm{PNN}^{+}$and $\mathrm{Kv} 3 \cdot 1^{+}$neuron densities were quantified as ipsilateral to contralateral ratios. $\mathbf{d}$ Changes in glutamatergic and GABAergic synapse densities were calculated as ipsilateral to contralateral ratios. Data are shown for each animal examined $(n \geq 5$ animals per condition). Data are medians (lines inside boxes)/ means (filled squares inside boxes) \pm interquartile ranges (IQR; boxes) with $10 / 90 \%$ ranks as whiskers. Open diamonds are data points. The asterisks indicate significant differences with control, based on KruskalWallis tests $(* * * p<0.001, * * p<0.01)$. ns not significant 
a Electrophysiological properties of fast-spiking inhibitory interneurons
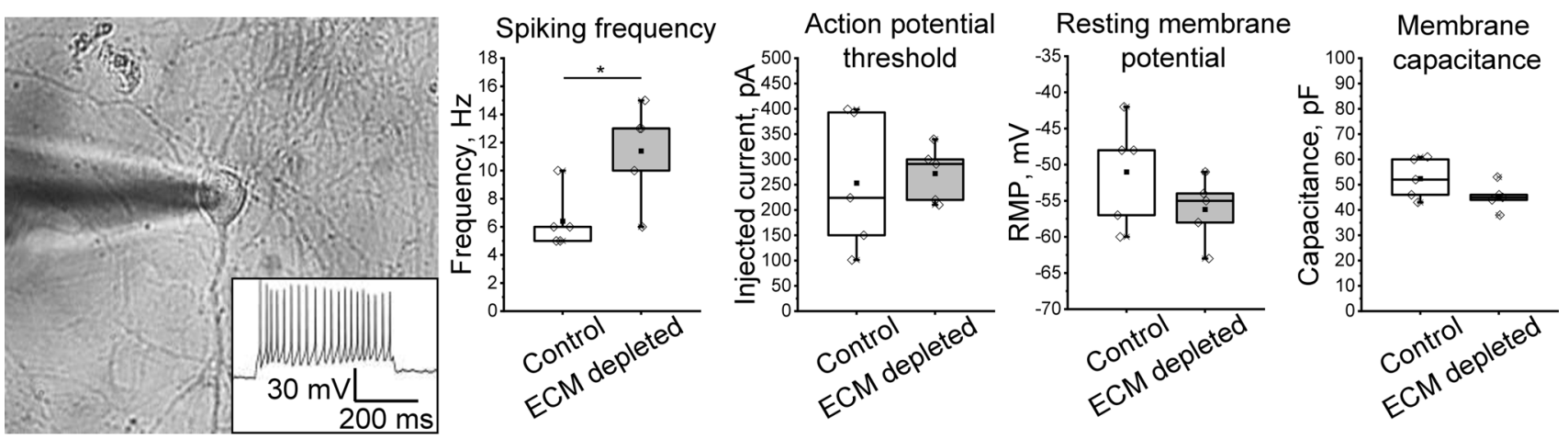

b Electrophysiological properties of excitatory pyramidal neurons
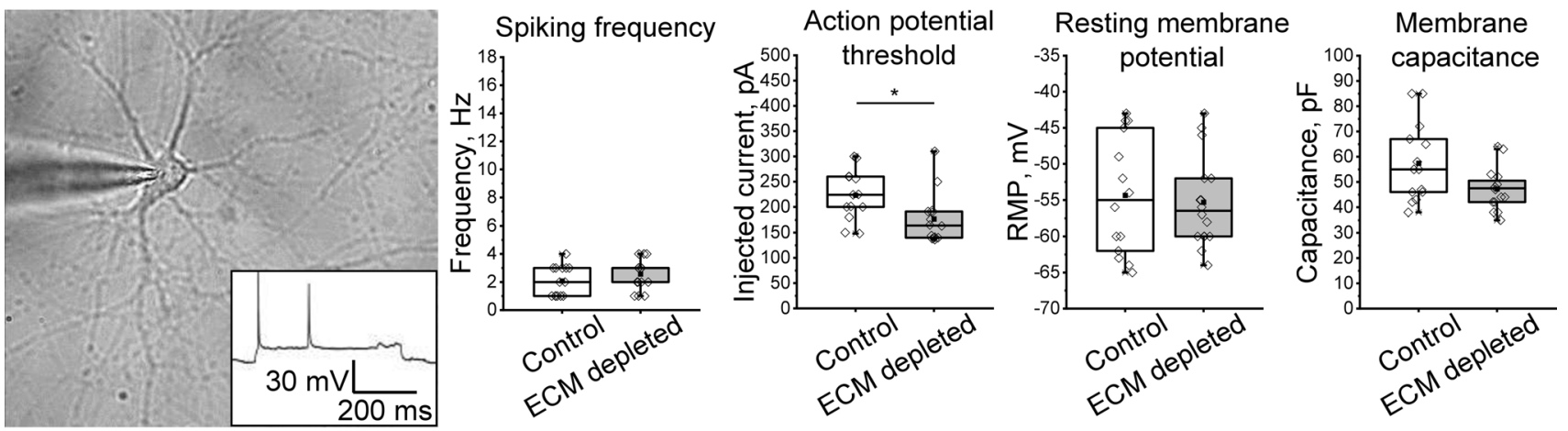

C Miniature inhibitory postsynaptic currents (mIPSCs)
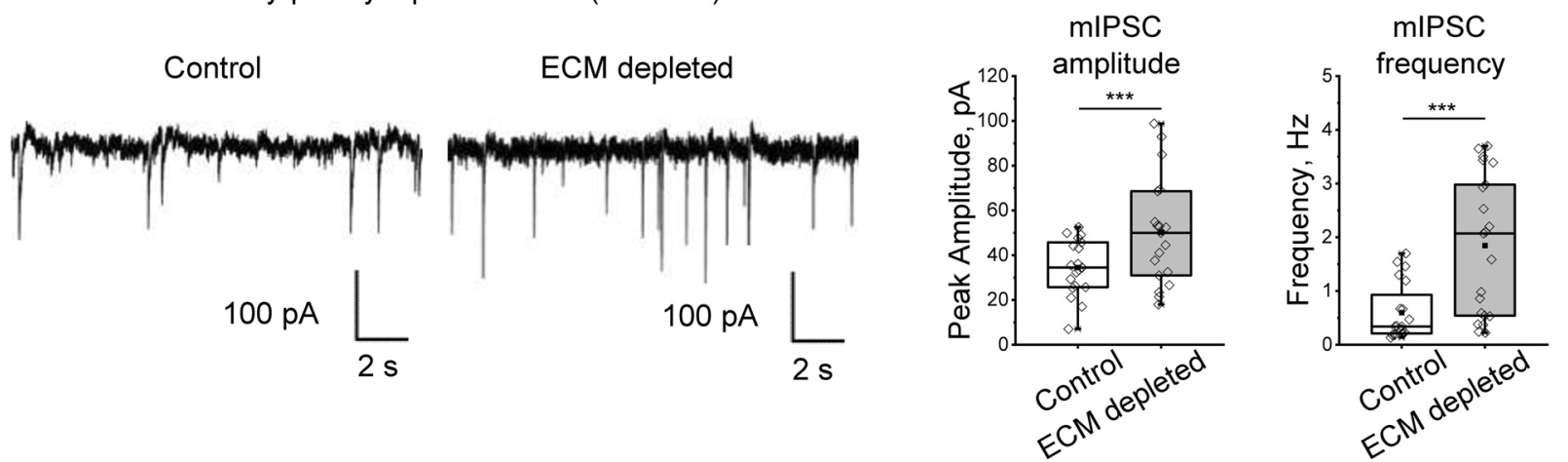

Fig. 3 ECM depletion affects electrophysiological properties in single neurons and strengthens inhibitory synapses. Spiking frequency, action potential threshold, resting membrane potential, and membrane capacitance were evaluated in the fast-spiking inhibitory interneurons (a) and the excitatory neurons (b). The results were obtained from 5 independent experiments. c Patch clamp recordings in presence of sodium channel blocker (TTX) and glutamate receptor antagonists (DNQX and D-AP5) reveal miniature inhibitory postsynaptic currents (mIPSCs). Representative current tracks exemplify mIPSCs detected

threshold, rendering these cells more excitable (Fig. 3b). Spiking frequency and passive membrane properties were not altered in excitatory neurons. in control and ECM depleted cultures. Quantifications of mIPSC amplitude and frequency indicate that ECM depletion increased the total inhibitory input to single neurons ( $n \geq 19$ neurons per condition, results obtained from 5 independent experiments). Data are medians (lines inside boxes)/ means (filled squares inside boxes) $\pm \mathrm{IQR}$ (boxes) with 10/ $90 \%$ ranks as whiskers. Open diamonds are data points. The asterisks indicate significant differences with control, based on Kruskal-Wallis tests $(* p<0.05, * * * p<0.001)$

\section{ECM depletion increases the strength of inhibitory synapses}

Since ECM depletion preferentially reduced the density of inhibitory synapses, we further asked whether the strength of inhibitory input to individual neurons is functionally altered. 

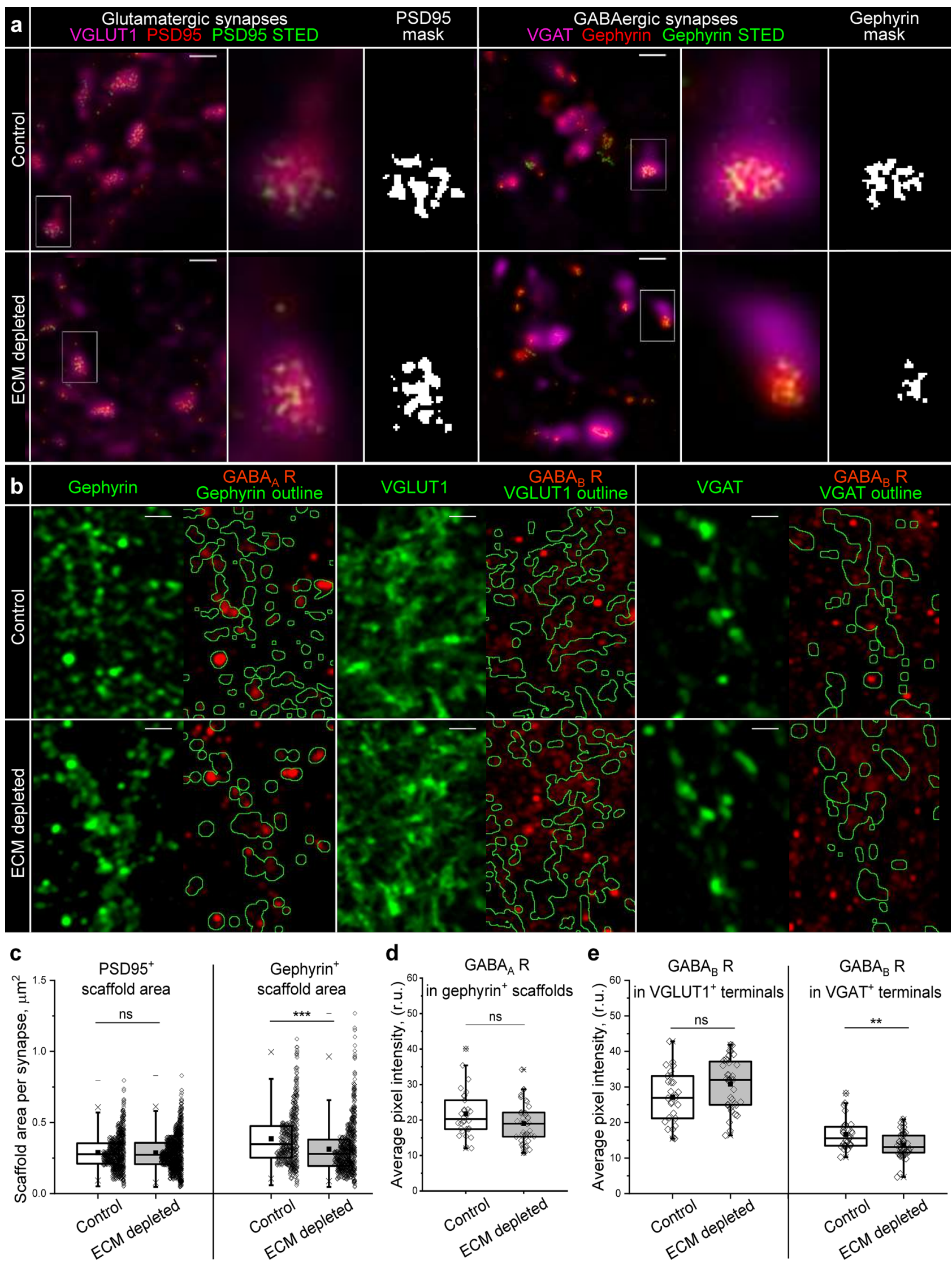

e

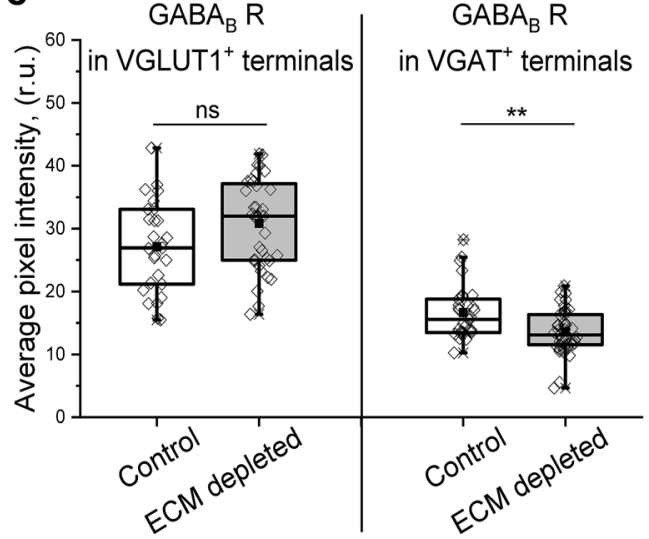


4Fig. 4 ECM depletion alters the pre- and postsynaptic organization of inhibitory synapses. a Stimulated emission depletion (STED) microscopy resolves the morphology of presynaptic scaffolds in glutamatergic and GABAergic synapses. Scale bars, $1 \mu \mathrm{m}$. Single synapses highlighted with white rectangles are magnified and the corresponding masks of postsynaptic scaffolds are shown. $\mathbf{b}$ The panel illustrates the analysis of $\mathrm{GABA}_{\mathrm{A}}$ receptor $\left(\mathrm{GABA}_{\mathrm{A}} \mathrm{R}\right)$ expression in inhibitory postsynapses (gephyrin ${ }^{+}$areas), $\mathrm{GABA}_{\mathrm{B}}$ receptor $\left(\mathrm{GABA}_{\mathrm{B}}\right.$ $\mathrm{R}$ ) expression in excitatory (VGLUT1 ${ }^{+}$areas) and inhibitory (VGAT ${ }^{+}$areas) postsynapses. The outlined areas (green) depict the regions in which the immunoreactivity of GABA receptors was measured. Scale bars, $2 \mu \mathrm{m}$. $\mathbf{c}$ The area of scaffolds containing PSD95 or gephyrin was quantified in single synapses $(n \geq 580$ synapses per condition, results from 5 independent experiments). d Immunoreactivity of $\mathrm{GABA}_{\mathrm{A}}$ receptors in GABAergic postsynapses. e Immunoreactivity of $\mathrm{GABA}_{\mathrm{B}}$ receptors in glutamatergic and GABAergic presynapses. d, e The average pixel intensity was quantified for each neuron examined ( $\mathrm{n} \geq 30$ cells per condition, results from 5 independent experiments). Data are medians (lines inside boxes)/ means (filled squares inside boxes) \pm IQR (boxes) with $10 / 90 \%$ ranks as whiskers. Open diamonds are data points. The asterisks indicate significant differences with control, based on Kruskal-Wallis tests $(* * * p<0.001$, $* * p<0.01)$. ns, not significant

Spontaneous neurotransmitter release in inhibitory synapses was measured by recording miniature inhibitory postsynaptic currents (mIPSCs) in mature neurons (Fig. 3c).

For recording mIPSCs, $1 \mu \mathrm{M}$ tetrodotoxin (TTX) was applied to prevent action potential-driven synaptic release, and a mixture of glutamate receptor antagonists $(10 \mu \mathrm{M}$ DNQX and $10 \mu \mathrm{M}$ D-APV) was added to isolate inhibitory currents. Interestingly, ECM depletion increased both amplitude and frequency (Fig. 3c, d) of mIPSCs. While the higher mIPSC amplitude indicates elevated neurotransmitter content per synaptic vesicle [27] or increased number and conductance of postsynaptic receptors [49], the higher frequency can result from increased release probability and the number of synapses [58]. Considering the reduced synapse number (Fig. 1c), these data show that ECM depletion increased the strength of inhibitory synapses. We suggest that this effect reflects a homeostatic mechanism for restoring the excitation-inhibition (E-I) balance in case of losing a portion of inhibitory synapses.

\section{ECM depletion reduces presynaptic expression of $\mathrm{GABA}_{B}$ receptors in inhibitory synapses}

To understand how ECM depletion facilitates inhibition, we examined the ultrastructural organization of inhibitory and excitatory postsynapses and analyzed the distribution of GABA receptors. We used stimulated emission depletion (STED) microscopy to uncover the morphology of gephyrin and PSD95 containing scaffolds within structurally complete GABAergic and glutamatergic synapses (Fig. 4a).

By analyzing the area of binary masks representing single synaptic scaffolds, we determined that ECM depletion reduced the size of gephyrin, but not PSD95 scaffolds
(Fig. 4c). Because gephyrin scaffolds are essential for the clusterization of postsynaptic $\mathrm{GABA}_{\mathrm{A}}$ receptors, we measured the immunoreactivity of $\mathrm{GABA}_{\mathrm{A}}$ receptors inside gephyrin containing postsynapses (Fig. 4b, d), but found no significant changes after ECM depletion. The total expression of $\mathrm{GABA}_{\mathrm{A}}$ receptors was not affected (Fig. S6).

$\mathrm{GABA}_{\mathrm{B}}$ receptors act as negative regulators of neurotransmitter release on the presynaptic side of both excitatory and inhibitory synapses. By measuring the immunoreactivity of $\mathrm{GABA}_{\mathrm{B}}$ receptors on $\mathrm{VGLUT}^{+}$and $\mathrm{VGAT}^{+}$presynaptic terminals, we revealed that ECM depletion preferentially reduces $\mathrm{GABA}_{\mathrm{B}}$ receptor expression in GABAergic presynapses (Fig. 4b, e). These results suggest that the increased strength of inhibitory synapses after ECM depletion is associated with reduced reciprocal inhibition of neurotransmitter release. Neither total nor postsynaptic expression of $\mathrm{GABA}_{\mathrm{B}}$ receptors was significantly influenced by ECM depletion (Figs. S6 and S7).

\section{ECM depletion increases neuronal activity and facilitates spiking-bursting transitions}

Knowing that ECM depletion reduces inhibitory connectivity, increases the strength of both excitatory and inhibitory synapses, elevates the firing frequency of fast-spiking inhibitory interneurons, and enhances the excitability of slower excitatory neurons, we asked how these opposing changes affect neuronal activity at the network level. Spontaneous network activity was investigated using multiple electrode arrays (MEAs). Within this methodology, neuronal cultures are grown on an array of electrodes, each detecting population spikes and bursts, generated by small groups of neurons (10-15 in our experiments), located within $100 \mu \mathrm{m}$ radius from the center of the electrode (Fig. 5a, b). While the frequency of spikes measured as the electrode mean firing rate (MFR) represents general neuronal activity, the network phase transitions are represented by bursting behavior, which was evaluated as mean bursting rate (MBR) changes. In mature networks, spiking-bursting transitions are mostly synchronized, as indicated by the alignment of burst events (Fig. 5c). Therefore, MBR measurements also partially reflect neuronal network synchrony.

We analyzed how ECM depletion alters neuronal activity and bursting behavior by measuring MFR and MBR changes after ChABC (500 mU/ml, $16 \mathrm{~h}$ ) or control (PBS, $16 \mathrm{~h}$ ) treatment. The activity recorded by single electrodes was compared to the baseline activity of the same electrode before treatment (Fig. 5d, e). On average, ECM depletion increased MFR by $69.8 \pm 5.2 \%$ (mean \pm s.e.m.) and MBR by $102.0 \pm 9.6 \%$ (mean \pm s.e.m.). The increase of neuronal network activity and facilitated bursting was inhibition-dependent since the blockage of $\mathrm{GABA}_{\mathrm{A}}$ receptors $(6 \mu \mathrm{M}$ bicuculline metiodide for $30 \mathrm{~min}$ ) levelled the effect of ECM depletion. 
a

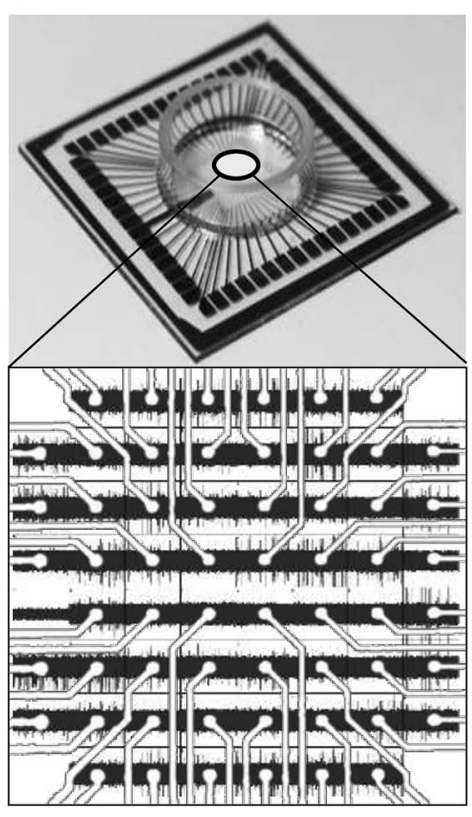

b

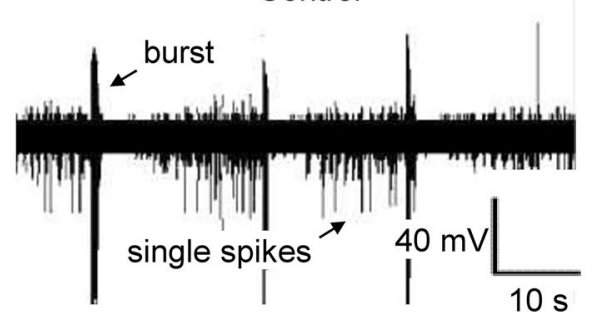

ECM depleted

C
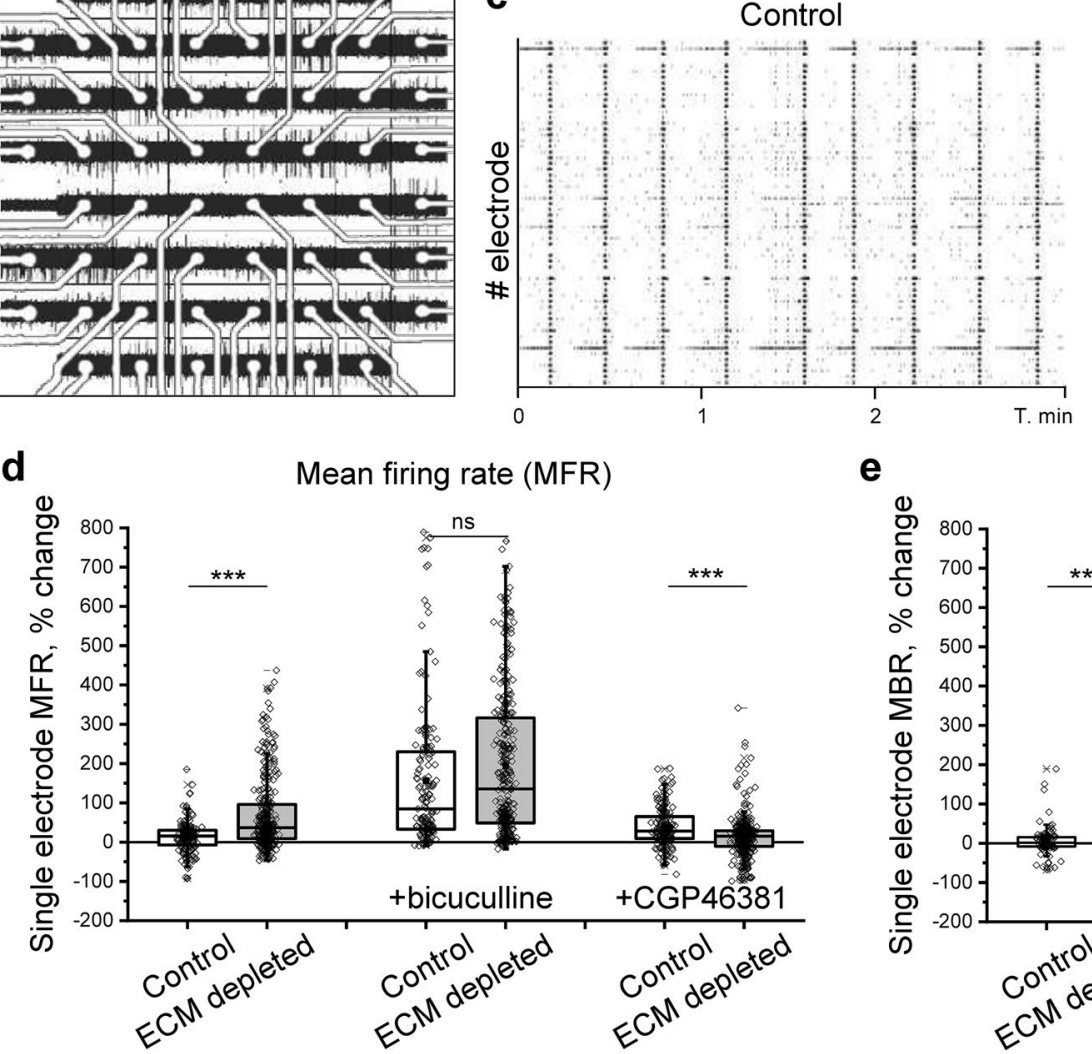

Fig. 5 The increase of neuronal network activity after ECM depletion is inhibition dependent. a Neuronal network activity was examined using multiple electrode arrays (MEAs). The panel demonstrates the layout and network activity recorded on a MEA chip with a square array of 59 electrodes. b Representative voltage tracks exemplify spikes and bursts detected by single electrodes in control and ECM depleted cultures. c Raster plots show synchronized network activity in control and ECM depleted cultures. Black ticks are single spikes, magenta bars are burst events. The changes of (d) mean firing rate (MFR) and (e) mean bursting rate (MBR) were quantified for sin-

The blockage of $\mathrm{GABA}_{\mathrm{B}}$ receptors (100 $\mu \mathrm{M}$ CGP46381 for $30 \mathrm{~min}$ ) moderately enhanced neuronal activity. After ECM depletion, the effect of $\mathrm{GABA}_{\mathrm{B}}$ receptor blockage was significantly reduced, as indicated by MFR quantification after treatment with CGP46381. Hence, the increase of neuronal network activity after ECM depletion functionally involved the downregulation of $\mathrm{GABA}_{\mathrm{B}}$ receptors.

e
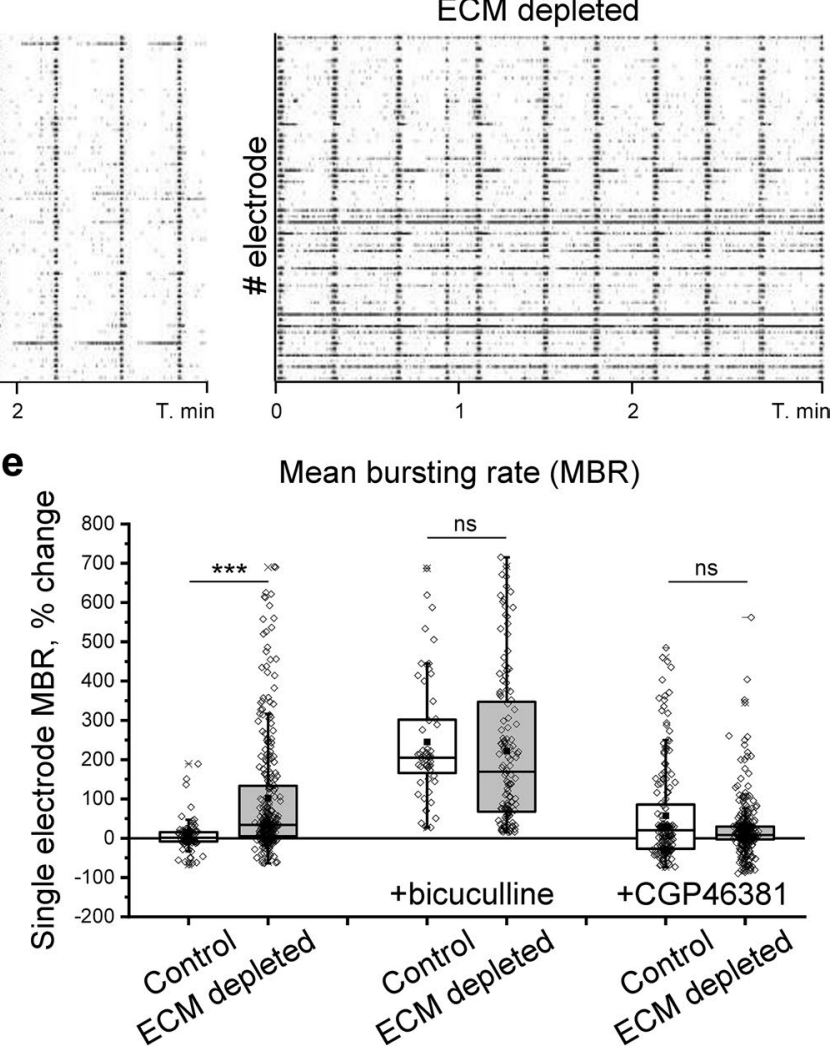

Mean bursting rate (MBR)

gle electrodes as the differences with the baseline activity of the same electrode before treatment $(n \geq 169$ electrodes per condition, results from 5 independent experiments). The effects of $\mathrm{GABA}_{\mathrm{A}}$ and $\mathrm{GABA}_{\mathrm{B}}$ receptor blockage were analyzed by comparing single electrode activity before and after incubation with the antagonist $(6 \mu \mathrm{M}$ bicuculline and $100 \mu \mathrm{M}$ CGP46381, respectively). Data are medians (lines inside boxes)/ means (filled squares inside boxes) $\pm \mathrm{IQR}$ (boxes) with 10/ 90\% ranks as whiskers. Open diamonds are data points. The asterisks indicate significant differences with control, based on Kruskal-Wallis tests $(* * * p<0.001)$. $n s$ not significant

\section{Loss of inhibitory synapses after ECM depletion renders inhibitory control inefficient}

The number and strength of GABAergic synapses are essential determinants of inhibitory input that control the activity and synchronization in neuronal networks. We revealed that these parameters undergo opposing changes after ECM 
a

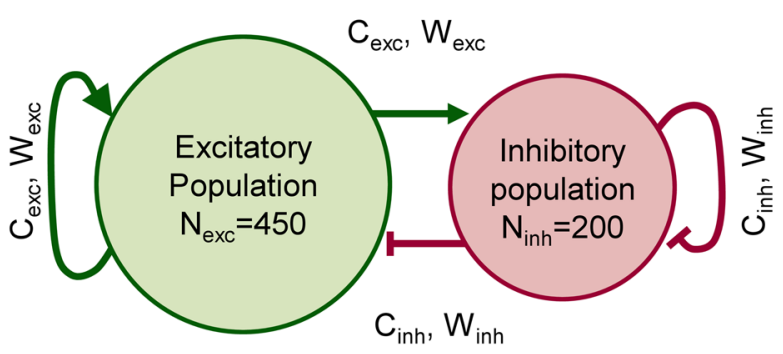

C

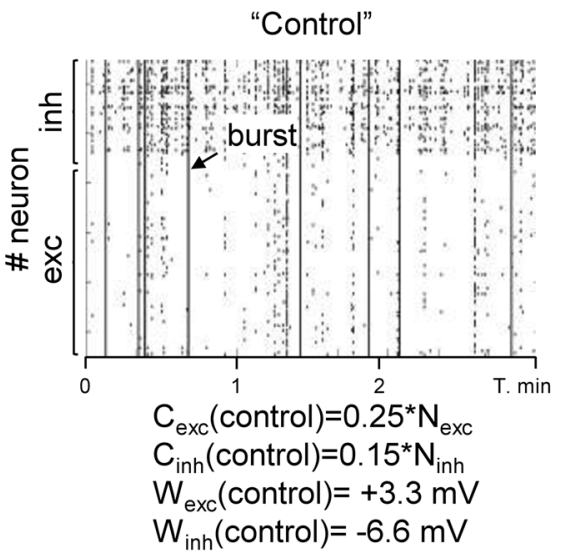

b
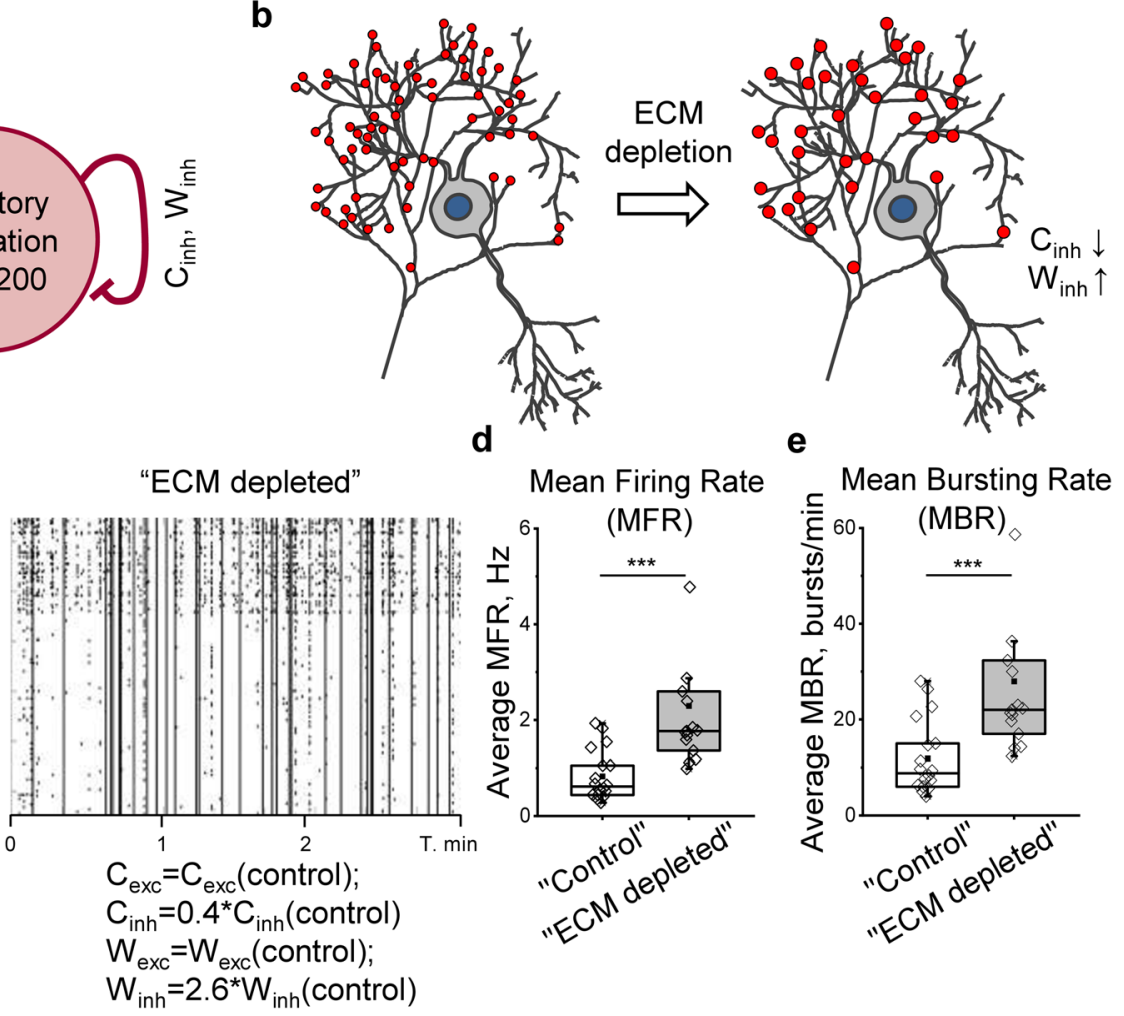

Mean Firing Rate

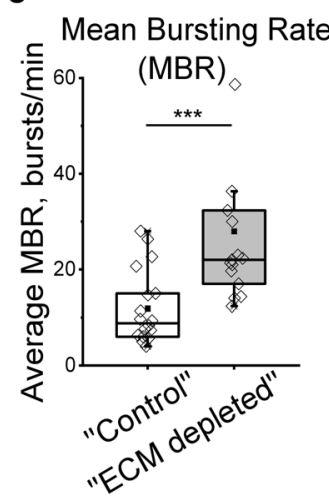

f
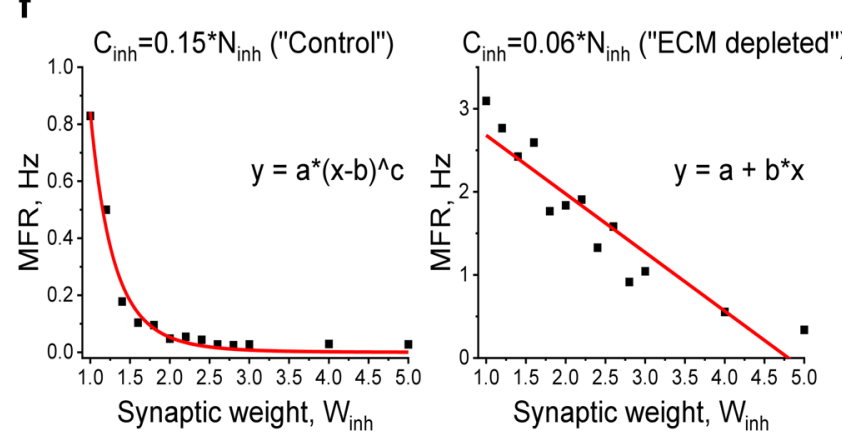

Fig. 6 Network activity simulation in silico indicates the prevailing role of inhibitory connectivity reduction following ECM depletion. a The schematic drawing illustrates the model of the spiking neuron network. $\mathrm{N}_{\text {inh }}$ and $\mathrm{N}_{\mathrm{exc}}$ are numbers of inhibitory and excitatory neurons, $\mathrm{W}_{\text {inh }}$ and $\mathrm{W}_{\text {exc }}$ are weights of corresponding synapses. b The ECM depletion was mimicked by tuning $\mathrm{C}_{\text {inh }}$ and $\mathrm{W}_{\text {inh }}$ parameters in accordance with experimentally observed changes. c Raster plots exemplify the activity of "control" and "ECM depleted" networks. The corresponding simulation parameters are depicted. Ticks are single spikes, vertical dashes indicate burst events. The quantification of network average (d) mean firing rate (MFR) and (e) mean bursting rate (MBR) is shown for "control" and "ECM depleted"

depletion, implying the necessity to understand their interaction at the network level. Using the earlier elaborated method [21], we reconstructed the observed alterations in silico (Fig. 6a). Excitatory and inhibitory connectivity was defined as the proportion of all neurons providing the input to a single cell $\left(\mathrm{C}_{\mathrm{exc}}=0.25^{*} \mathrm{~N}_{\mathrm{exc}}, \mathrm{C}_{\mathrm{inh}}=0.15^{*} \mathrm{~N}_{\mathrm{inh}}\right.$ in

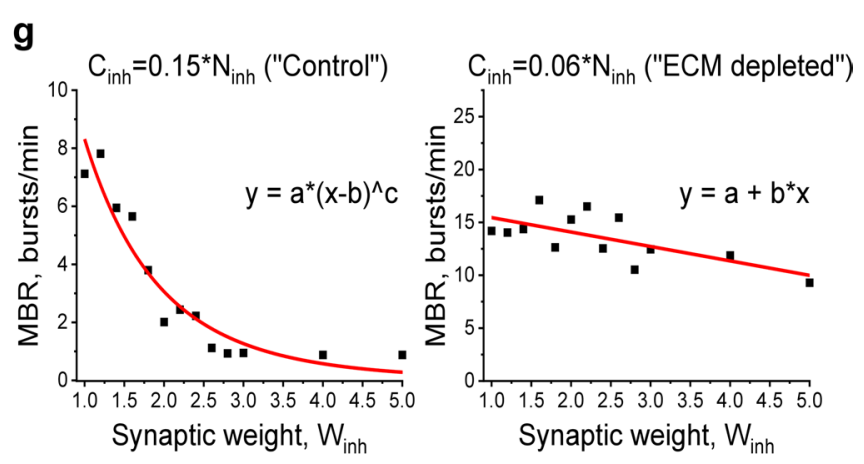

simulation conditions. Data are medians (lines inside boxes)/ means (filled squares inside boxes) \pm IQR (boxes) with $10 / 90 \%$ ranks as whiskers. Open diamonds are data points. The asterisks indicate significant differences with the control, based on Kruskal-Wallis tests $(* * * p<0.001)$. (f) MFR and (g) MBR are quantified in a range of $\mathrm{W}_{\text {inh }}$ changes for "control" and "ECM depleted" inhibitory connectivity. Note that the reduction of inhibitory connectivity switches the dependence of network activity on inhibitory synapse weight from power law to linearity. Squares indicate the mean of simulation repetitions, fit functions are shown in red. For each condition, 15 independent simulation experiments were performed

"control"), based on the previous study [41] and connectivity estimations in neuronal cultures [51]. The strength of single connections was defined by synaptic weights reflecting membrane potential changes upon synapse activation ( $\mathrm{W}_{\mathrm{exc}}=+3.3 \mathrm{mV}, \mathrm{W}_{\mathrm{inh}}=-6.6 \mathrm{mV}$ in "control"). Thereby, excitation and inhibition were balanced, and the 
computations were performed in a near-critical state (Fig. S8) characterized by stable spiking-bursting transitions. ECM depletion was simulated by modifying inhibitory connectivity $\left(\mathrm{C}_{\text {inh }}\right)$ and synaptic weights $\left(\mathrm{W}_{\text {inh }}\right)$ in accordance with experimentally observed changes (Fig. 6b). In agreement with in vitro experiments, ECM depletion increased network MFR (Fig. 6c, d) and bursting rates (Fig. 6c, e). Of note, our approach closely resembled the intrinsic variability in real neuronal networks, because the connectivity matrix was newly generated for each simulation instance.

To compare the impact of $\mathrm{C}_{\mathrm{inh}}$ and $\mathrm{W}_{\mathrm{inh}}$ on the resulting neuronal activity, we measured MFR and MBR over a range of different $\mathrm{C}_{\text {inh }}$ and $\mathrm{W}_{\text {inh }}$ values (Fig. S9). Excitatory input parameters $\mathrm{C}_{\mathrm{exc}}$ and $\mathrm{W}_{\mathrm{exc}}$ were set to control values. The reduction of $\mathrm{C}_{\mathrm{inh}}$ negatively and linearly correlated with network MFR $(r=-0.98 ; p<0.01)$ and MBR $(r=-0.92 ; p<0.01)$, while the increased $\mathrm{C}_{\mathrm{inh}}$ strongly diminished neuronal activity. Under moderate decrease of $\mathrm{C}_{\mathrm{inh}}\left(\mathrm{C}_{\mathrm{inh}}=0.125 * \mathrm{~N}_{\mathrm{inh}}\right.$ and $\left.\mathrm{C}_{\mathrm{inh}}=0.1 * \mathrm{~N}_{\mathrm{inh}}\right)$, increasing $\mathrm{W}_{\text {inh }}$ partially compensated MFR and MBR changes. However, when $\mathrm{C}_{\mathrm{inh}}$ was set in accordance with ECM depletion effect in vitro ( $40 \%$ of control, $\mathrm{C}_{\mathrm{inh}}=0.06 * \mathrm{~N}_{\mathrm{inh}}$ ), both MFR and MBR remained elevated in a broad range of $\mathrm{W}_{\text {inh }}$. While changing inhibitory connectivity from $\mathrm{C}_{\text {inh }}=0.15^{*} \mathrm{~N}_{\text {inh }}$ in "control" simulation to $\mathrm{C}_{\text {inh }}=0.06 * \mathrm{~N}_{\text {inh }}$ in "ECM depleted" simulation, the dependence of MFR and MBR on $\mathrm{W}_{\text {inh }}$ switched from a power law to linearity (Fig. 6f, g). Conclusively, the reduction of inhibitory connectivity after ECM depletion rendered inhibitory control inefficient and increased the resulting activity of neuronal networks.

\section{Discussion}

Here we demonstrate that the brain ECM supports the maintenance of neuronal network E-I balance by retaining inhibitory connectivity. ECM depletion preferentially decreases the density of inhibitory synapses and the size of inhibitory postsynaptic scaffolds, while it homeostatically increases inhibitory synapse strength. Commonly, inhibitory synapse scaling downregulates neuronal network activity and synchronization, providing a key mechanism for neuronal activity adjustment [65]. After ECM depletion, the degree of inhibitory connectivity reduces to an extent that inhibitory synapse scaling is no longer efficient in controlling the state of neuronal networks. As a result, neuronal network activity and synchrony increase.

We observed that $\sim 60 \%$ of GABAergic synapses are lost already $16 \mathrm{~h}$ after ECM depletion. How could such a major decrease in synapse density occur without increased neuronal cell death? Recent findings indicate that neuronal networks are continuously remodelled and that synapses dynamically wane and re-emerge within a few days [52].
Inhibitory synapses are especially dynamic, about $60 \%$ of them retract and return at the same place within 4 days [71]. Intuitively, this structural volatility but spatial persistency implies the existence of a stable framework to secure the integrity of neuronal circuits. Our data suggest that the brain ECM provides such a framework to stabilize inhibitory connectivity. After ECM depletion, the reduction of GABAergic synapse density may be due to the increased volatility and inability of synaptic boutons to find their postsynaptic site. The demarcation of postsynaptic sites has been proposed to be defined by PNNs [26, 63], the facet-like ECM coatings that compartmentalize neuronal surface. In mice lacking several ECM glycoproteins, the defective formation of PNNs associated with an increased ratio of excitatory to inhibitory synapses [34]. PNNs covering parvalbumincontaining interneurons maintain orthodenticle homeobox 2 (Otx2) dependent breaks on adult brain plasticity [2,5]. We hypothesize that a less dense ECM layer may play a similar role on neurons devoid of PNNs.

At the level of single synapses, ECM depletion increases inhibitory synapse strength, as indicated by the increased mIPSC amplitude and frequency together with the decreased synapse density. With STED microscopy, we observed the reduced size of postsynaptic gephyrin scaffolds after ECM depletion. However, despite the known role of gephyrin for postsynaptic $\mathrm{GABA}_{\mathrm{A}}$ receptor clustering and stabilization [15], neither expression nor localization of $\mathrm{GABA}_{\mathrm{A}}$ receptors was affected. Apparently, the gephyrin-containing scaffolds condensed and retained $\mathrm{GABA}_{\mathrm{A}}$ receptors. On the presynaptic side, we found a significant reduction of $\mathrm{GABA}_{\mathrm{B}}$ receptor expression on $\mathrm{VGAT}^{+}$terminals, which functionally associated with the diminished sensitivity to the $\mathrm{GABA}_{\mathrm{B}}$ antagonist CGP46381. Presynaptic GABA ${ }_{\mathrm{B}}$ receptors act as activity-dependent regulators of neurotransmitter release. Upon repetitive stimulation, the spillover of GABA activates presynaptic $\mathrm{GABA}_{\mathrm{B}}$ receptors, transiently reducing subsequent neurotransmitter release $[18,53]$. Our results indicate that ECM depletion attenuated this mechanism and increased inhibitory synapse strength via presynaptic facilitation. It remains unclear how exactly the ECM regulates $\mathrm{GABA}_{\mathrm{B}}$ receptor localization and function. The extracellular complement control protein module CCP1 of GABA $\mathrm{R} 1$ subunit interacts with laminin $\alpha 5$ subunit and fibulin-2 of the ECM [8, 55], but the functional consequences of this interaction need further investigation.

Our data shows that the increased synaptic strength does fully compensate for the reduction of inhibitory synapse number after ECM depletion. As a result, the reduction of inhibitory connectivity outweighs the increased inhibitory synapse strength, and the E-I balance switches towards excitation. Thereby, ECM depletion increases neuronal activity and network synchrony, as evidenced by MEA recordings and in silico simulations. A similar increase of network 
activity could arise from the reduced excitatory input in fastspiking interneurons, as observed in the hippocampus after ECM depletion [37], and the decreased rate of their spontaneous firing, as observed in a model of peritumoral epilepsy [67]. In this work, we observed neither significant changes of excitability [37] nor increased excitatory input [24] in fast-spiking interneurons, which were previously described in a similar model of ECM depletion. Unlike the VGLUT2 ${ }^{+}$ thalamic inputs in the visual cortex [24, 48], ECM depletion does not significantly alter the local excitatory connectivity in somatosensory cortex layers $3-5$, as indicated by the $\mathrm{VGLUT}^{+}$synapse quantifications. Although the possibility of increased stimulation of GABAergic interneurons via the long-range thalamocortical projections remains, our results indicate that inhibitory synapse stabilization is a key mechanism by which ECM supports E-I balance in local neuronal networks. In addition, E-I balance changes after ECM depletion may be further amplified by the increased excitability of excitatory neurons that we observed. However, the reduction of action potential threshold in excitatory neurons can be counterbalanced by the increased firing rate of inhibitory interneurons, which we also detected experimentally. The mechanism by which ECM depletion modifies electrophysiological properties of individual neurons potentially involves altered buffering of membrane-associated calcium [70], but remains to be further explored.

Altered E-I balance following the ECM breakdown is likely a key component of the pathophysiology of psychosis $[50,64]$ and epilepsy $[1,67]$. In ischemic stroke, adjusting the E-I balance after ECM decomposition may, on the contrary, support neurological recovery. Post-stroke neuroplasticity is impaired by decreased neuronal excitability in perilesional brain areas $[17,73]$. In light of the new evidence we present here, the transient decline in cortical ECM integrity after ischemia [22] may support neuronal network rewiring by stimulating neuronal activity. Hence, the controlled degradation of ECM could be a promising target in stroke therapy, as it may allow promoting neuronal activity and plasticity.

The therapeutic potential of ECM degradation in the injured brain will crucially depend on the precise targeting of ECM modifications. The crude ablation of the ECM elicits memory loss and learning deficits [40, 62]. In this study, we demonstrate that the near-complete ECM digestion disrupts criticality in neuronal networks, indicated by the switch of dependence between neuronal activity and inhibitory synapse strength from power law to linearity. The dynamic tuning of cortical circuits to criticality is essential for efficient information processing in the brain [30, 42, 45]. Refined tools for controlled ECM decomposition will not only expand opportunities for research but will also open new directions in neurorestorative therapies.
Supplementary Information The online version contains supplementary material available at https://doi.org/10.1007/s00018-021-03861-3.

Acknowledgements The German Research Foundation (DFG), grants HE3173/11-1, 12-1 and 13-1 to D.M.H., supported this work. For the maintenance of microscopy equipment and ensuring the high quality of imaging, the authors are grateful to Dr. Anthony Squire (IMCES Essen). For providing the MatLab script for MEA analysis, the authors sincerely thank Dr. Michela Chiappalone.

Author contributions E.D. and D.M.H. planned and designed the study. E.D., M.F., D.M-C. and M.B. performed experiments and analyzed data. E.D. and D.M.H. drafted the manuscript. A.F. and D.M.H. provided essential advice on experimental design and contributed materials. All authors discussed the results and contributed to the final manuscript.

Funding Open Access funding enabled and organized by Projekt DEAL. The German Research Foundation (DFG), grants 259317790 , 389030878 and 405358801 to D.M.H., and 290189690 to A.F., supported this work.

Data availability All data related to this work is presented in the paper and its supplements. Raw data is available at https://doi.org/10.5061/ dryad.vx0k6djpp. The materials used in this study are available to any qualified researcher upon reasonable request addressed to D.M.H.

Code availability The code for network activity simulations is provided as a supplement to this paper. This code can be freely modified or reused for non-commercial purposes if appropriately cited. The Synapse counter plugin is available at https://github.com/SynPuCo/SynapseCou nter. All minor codes for image analysis are available upon reasonable request addressed to E.D.

\section{Declarations}

Conflicts of interest The authors declare no conflict of interest.

Ethical approval Experimental procedures were approved by the local government (Bezirksregierung Düsseldorf) and conducted in accordance to European Union (Directive 2010/63/EU) guidelines for the care and use of laboratory animals.

Open Access This article is licensed under a Creative Commons Attribution 4.0 International License, which permits use, sharing, adaptation, distribution and reproduction in any medium or format, as long as you give appropriate credit to the original author(s) and the source, provide a link to the Creative Commons licence, and indicate if changes were made. The images or other third party material in this article are included in the article's Creative Commons licence, unless indicated otherwise in a credit line to the material. If material is not included in the article's Creative Commons licence and your intended use is not permitted by statutory regulation or exceeds the permitted use, you will need to obtain permission directly from the copyright holder. To view a copy of this licence, visit http://creativecommons.org/licenses/by/4.0/.

\section{References}

1. Arranz AM, Perkins KL, Irie F, Lewis DP, Hrabe J, Xiao F, Itano N, Kimata K, Hrabetova S, Yamaguchi Y (2014) Hyaluronan deficiency due to Has3 knock-out causes altered neuronal activity 
and seizures via reduction in brain extracellular space. J Neurosci 34:6164-6176

2. Bavelier D, Levi DM, Li RW, Dan Y, Hensch TK (2010) Removing brakes on adult brain plasticity: from molecular to behavioral interventions. J Neurosci 30:14964-14971

3. Beggs JM, Klukas J, Chen W (2007) Connectivity and Dynamics in Local Cortical Networks. In: Jirsa VK, McIntosh AR (eds) Handbook of Brain Connectivity. Springer, Berlin, pp 91-116

4. Berretta S, Pantazopoulos H, Markota M, Brown C, Batzianouli ET (2015) Losing the sugar coating: potential impact of perineuronal net abnormalities on interneurons in schizophrenia. Schizophr Res 167:18-27

5. Beurdeley M, Spatazza J, Lee HH, Sugiyama S, Bernard C, Di Nardo AA, Hensch TK, Prochiantz A (2012) Otx2 binding to perineuronal nets persistently regulates plasticity in the mature visual cortex. J Neurosci 32:9429-9437

6. Bhatia A, Moza S, Bhalla US (2019) Precise excitation-inhibition balance controls gain and timing in the hippocampus. Life 8:e43415

7. Bikbaev A, Frischknecht R, Heine M (2015) Brain extracellular matrix retains connectivity in neuronal networks. Sci Rep 5:14527

8. Blein S, Ginham R, Uhrin D, Smith BO, Soares DC, Veltel S, McIlhinney RA, White JH, Barlow PN (2004) Structural analysis of the complement control protein (CCP) modules of GABA(B) receptor 1a: only one of the two CCP modules is compactly folded. J Biol Chem 279:48292-48306

9. Bologna LL, Pasquale V, Garofalo M, Gandolfo M, Baljon PL, Maccione A, Martinoia S, Chiappalone M (2010) Investigating neuronal activity by SPYCODE multi-channel data analyzer. Neural Netw 23:685-697

10. Carulli D, Broersen R, de Winter F, Muir EM, Meskovic M, de Waal M, de Vries S, Boele HJ, Canto CB, De Zeeuw CI, Verhaagen J (2020) Cerebellar plasticity and associative memories are controlled by perineuronal nets. Proc Natl Acad Sci USA 117:6855-6865

11. Carulli D, Rhodes KE, Brown DJ, Bonnert TP, Pollack SJ, Oliver K, Strata P, Fawcett JW (2006) Composition of perineuronal nets in the adult rat cerebellum and the cellular origin of their components. J Comp Neurol 494:559-577

12. Carulli D, Rhodes KE, Fawcett JW (2007) Upregulation of aggrecan, link protein 1, and hyaluronan synthases during formation of perineuronal nets in the rat cerebellum. J Comp Neurol 501:83-94

13. Chiu CQ, Barberis A, Higley MJ (2019) Preserving the balance: diverse forms of long-term GABAergic synaptic plasticity. Nat Rev Neurosci 20:272-281

14. Choi S-Y (2018) Synaptic and circuit development of the primary sensory cortex. Exp Mol Med 50:13

15. Choii G, Ko J (2015) Gephyrin: a central GABAergic synapse organizer. Exp Mol Med 47:e158

16. Chu P, Abraham R, Budhu K, Khan U, De Marco Garcia N, Brumberg JC (2018) The impact of perineuronal net digestion using chondroitinase $\mathrm{ABC}$ on the intrinsic physiology of cortical neurons. Neuroscience 388:23-35

17. Clarkson AN, Huang BS, Macisaac SE, Mody I, Carmichael ST (2010) Reducing excessive GABA-mediated tonic inhibition promotes functional recovery after stroke. Nature 468:305-309

18. Davies CH, Davies SN, Collingridge GL (1990) Paired-pulse depression of monosynaptic GABA-mediated inhibitory postsynaptic responses in rat hippocampus. J Physiol 424:513-531

19. Dityatev A, Bruckner G, Dityateva G, Grosche J, Kleene R, Schachner M (2007) Activity-dependent formation and functions of chondroitin sulfate-rich extracellular matrix of perineuronal nets. Dev Neurobiol 67:570-588

20. Dzyubenko E, Gottschling C, Faissner A (2016) Neuron-glia interactions in neural plasticity: contributions of neural extracellular matrix and perineuronal nets. Neural Plast 2016:5214961
21. Dzyubenko E, Juckel G, Faissner A (2017) The antipsychotic drugs olanzapine and haloperidol modify network connectivity and spontaneous activity of neural networks in vitro. Sci Rep 7:11609

22. Dzyubenko E, Manrique-Castano D, Kleinschnitz C, Faissner A, Hermann DM (2018) Topological remodeling of cortical perineuronal nets in focal cerebral ischemia and mild hypoperfusion. Matrix Biol 74:121-132

23. Dzyubenko E, Rozenberg A, Hermann DM, Faissner A (2016) Colocalization of synapse marker proteins evaluated by STEDmicroscopy reveals patterns of neuronal synapse distribution in vitro. J Neurosci Methods 273:149-159

24. Faini G, Aguirre A, Landi S, Lamers D, Pizzorusso T, Ratto GM, Deleuze C, Bacci A (2018) Perineuronal nets control visual input via thalamic recruitment of cortical PV interneurons. Life 7:e41520

25. Fares T, Stepanyants A (2009) Cooperative synapse formation in the neocortex. Proc Natl Acad Sci 106:16463-16468

26. Fawcett JW, Oohashi T, Pizzorusso T (2019) The roles of perineuronal nets and the perinodal extracellular matrix in neuronal function. Nat Rev Neurosci 20:451-465

27. Frerking M, Borges S, Wilson M (1995) Variation in GABA mini amplitude is the consequence of variation in transmitter concentration. Neuron 15:885-895

28. Frischknecht R, Heine M, Perrais D, Seidenbecher CI, Choquet D, Gundelfinger ED (2009) Brain extracellular matrix affects AMPA receptor lateral mobility and short-term synaptic plasticity. Nat Neurosci 12:897-904

29. Gandolfi D, Bigiani A, Porro CA, Mapelli J (2020) Inhibitory plasticity: from molecules to computation and beyond. Int J Mol Sci 21:1805

30. Gautam SH, Hoang TT, McClanahan K, Grady SK, Shew WL (2015) Maximizing sensory dynamic range by tuning the cortical state to criticality. PLoS Comput Biol 11:e1004576

31. Geissler M, Faissner A (2012) A new indirect co-culture set up of mouse hippocampal neurons and cortical astrocytes on microelectrode arrays. J Neurosci Methods 204:262-272

32. Geissler M, Gottschling C, Aguado A, Rauch U, Wetzel HC, Hatt H, Faissner A (2013) Primary hippocampal neurons, which lack four crucial extracellular matrix molecules, display abnormalities of synaptic structure and function and severe deficits in perineuronal net formation. J Neurosci 33:7742-7755

33. Gottschling C, Dzyubenko E, Geissler M, Faissner A (2016) The indirect neuron-astrocyte coculture assay: an in vitro set-up for the detailed investigation of neuron-glia interactions. J Vis Exp 117:e54757

34. Gottschling C, Wegrzyn D, Denecke B, Faissner A (2019) Elimination of the four extracellular matrix molecules tenascin-C, tenascin-R, brevican and neurocan alters the ratio of excitatory and inhibitory synapses. Sci Rep 9:13939

35. Haider B, Duque A, Hasenstaub AR, McCormick DA (2006) Neocortical network activity in vivo is generated through a dynamic balance of excitation and inhibition. J Neurosci 26:4535-4545

36. Härtig W, Mages B, Aleithe S, Nitzsche B, Altmann S, Barthel H, Krueger M, Michalski D (2017) Damaged neocortical perineuronal nets due to experimental focal cerebral ischemia in mice, rats and sheep. Front Integ Neurosci 11:15

37. Hayani H, Song I, Dityatev A (2018) Increased excitability and reduced excitatory synaptic input into fast-spiking CA2 interneurons after enzymatic attenuation of extracellular matrix. Front Cell Neurosci 12:149-149

38. Hendry S, Schwark H, Jones E, Yan J (1987) Numbers and proportions of GABA-immunoreactive neurons in different areas of monkey cerebral cortex. J Neurosci 7:1503-1519

39. Ho VM, Lee J-A, Martin KC (2011) The cell biology of synaptic plasticity. Science 334:623-628 
40. Hylin MJ, Orsi SA, Moore AN, Dash PK (2013) Disruption of the perineuronal net in the hippocampus or medial prefrontal cortex impairs fear conditioning. Learn Mem 20:267-273

41. Izhikevich EM (2003) Simple model of spiking neurons. IEEE Trans Neural Netw 14:1569-1572

42. Kinouchi O, Copelli M (2006) Optimal dynamical range of excitable networks at criticality. Nat Phys 2:348-351

43. Krishnaswamy VR, Benbenishty A, Blinder P, Sagi I (2019) Demystifying the extracellular matrix and its proteolytic remodeling in the brain: structural and functional insights. Cell Mol Life Sci 76:3229-3248

44. Lake EMR, Bazzigaluppi P, Mester J, Thomason LAM, Janik R, Brown M, McLaurin J, Carlen PL, Corbett D, Stanisz GJ, Stefanovic B (2017) Neurovascular unit remodelling in the subacute stage of stroke recovery. Neuroimage 146:869-882

45. Ma Z, Turrigiano GG, Wessel R, Hengen KB (2019) Cortical circuit dynamics are homeostatically tuned to criticality in vivo. Neuron 104:655-664 e654

46. Milior G, Di Castro MA, Sciarria LP, Garofalo S, Branchi I, Ragozzino D, Limatola C, Maggi L (2016) Electrophysiological properties of CA1 pyramidal neurons along the longitudinal axis of the mouse hippocampus. Sci Rep 6:38242

47. Mongillo G, Rumpel S, Loewenstein Y (2018) Inhibitory connectivity defines the realm of excitatory plasticity. Nat Neurosci 21:1463-1470

48. Nahmani M, Erisir A (2005) VGluT2 immunochemistry identifies thalamocortical terminals in layer 4 of adult and developing visual cortex. J Comp Neurol 484:458-473

49. Nusser Z, Cull-Candy S, Farrant M (1997) Differences in synaptic GABAA receptor number underlie variation in GABA mini amplitude. Neuron 19:697-709

50. Pantazopoulos H, Markota M, Jaquet F, Ghosh D, Wallin A, Santos A, Caterson B, Berretta S (2015) Aggrecan and chondroitin6-sulfate abnormalities in schizophrenia and bipolar disorder: a postmortem study on the amygdala. Transl Psychiatry 5:e496

51. Pastore VP, Massobrio P, Godjoski A, Martinoia S (2018) Identification of excitatory-inhibitory links and network topology in large-scale neuronal assemblies from multi-electrode recordings. PLOS Comp Biol 14:e1006381

52. Pfeiffer T, Poll S, Bancelin S, Angibaud J, Inavalli VK, Keppler K, Mittag M, Fuhrmann M, Nagerl UV (2018) Chronic 2P-STED imaging reveals high turnover of dendritic spines in the hippocampus in vivo. Elife 7:e34700

53. Pitler TA, Alger BE (1994) Differences between presynaptic and postsynaptic GABAB mechanisms in rat hippocampal pyramidal cells. J Physiol 72:2317-2327

54. Pizzorusso T, Medini P, Berardi N, Chierzi S, Fawcett JW, Maffei L (2002) Reactivation of ocular dominance plasticity in the adult visual cortex. Science 298:1248-1251

55. Pless E (2009) An investigation of interactions with extracellular matrix proteins mediated by the CCP modules of the metabotropic GABAB receptor. PhD Thesis, The University of Edinburgh, Scotland. http://hdl.handle.net/1842/5714

56. Pyka M, Wetzel C, Aguado A, Geissler M, Hatt H, Faissner A (2011) Chondroitin sulfate proteoglycans regulate astrocytedependent synaptogenesis and modulate synaptic activity in primary embryonic hippocampal neurons. Eur J Neurosci 33:2187-2202

57. Que L, Lukacsovich D, Luo W, Földy C (2021) Transcriptional and morphological profiling of parvalbumin interneuron subpopulations in the mouse hippocampus. Nat Commun 12:108

58. Roberto M, Madamba SG, Stouffer DG, Parsons LH, Siggins GR (2004) Increased GABA release in the central amygdala of ethanol-dependent rats. J Neurosci 24:10159-10166
59. Roll L, Faissner A (2014) Influence of the extracellular matrix on endogenous and transplanted stem cells after brain damage. Front Cell Neurosci 8:219

60. Salesse C, Charest J, Doucet-Beaupré H, Castonguay A-M, Labrecque S, De Koninck P, Lévesque M (2020) Opposite control of excitatory and inhibitory synapse formation by Slitrk2 and Slitrk5 on dopamine neurons modulates hyperactivity behavior. Cell Rep 30:2374-2386.e2375

61. Sederberg A, Nemenman I (2020) Randomly connected networks generate emergent selectivity and predict decoding properties of large populations of neurons. PLOS Comp Biol 16:e1007875

62. Shi W, Wei X, Wang X, Du S, Liu W, Song J, Wang Y (2019) Perineuronal nets protect long-term memory by limiting activitydependent inhibition from parvalbumin interneurons. Proc Natl Acad Sci 116:27063-27073

63. Sigal YM, Bae H, Bogart LJ, Hensch TK, Zhuang X (2019) Structural maturation of cortical perineuronal nets and their perforating synapses revealed by superresolution imaging. Proc Natl Acad Sci 116:7071-7076

64. Soleman S, Filippov MA, Dityatev A, Fawcett JW (2013) Targeting the neural extracellular matrix in neurological disorders. Neuroscience 253:194-213

65. Sprekeler H (2017) Functional consequences of inhibitory plasticity: homeostasis, the excitation-inhibition balance and beyond. Curr Opin Neurobiol 43:198-203

66. Tamamaki N, Yanagawa Y, Tomioka R, Miyazaki J-I, Obata K, Kaneko T (2003) Green fluorescent protein expression and colocalization with calretinin, parvalbumin, and somatostatin in the GAD67-GFP knock-in mouse. J Comp Neurol 467:60-79

67. Tewari BP, Chaunsali L, Campbell SL, Patel DC, Goode AE, Sontheimer H (2018) Perineuronal nets decrease membrane capacitance of peritumoral fast spiking interneurons in a model of epilepsy. Nat Commun 9:4724

68. Ting JT, Kalmbach B, Chong P, de Frates R, Keene CD, Gwinn RP, Cobbs C, Ko AL, Ojemann JG, Ellenbogen RG, Koch C, Lein E (2018) A robust ex vivo experimental platform for moleculargenetic dissection of adult human neocortical cell types and circuits. Sci Rep 8:8407

69. Trapp P, Echeveste R, Gros C (2018) E-I balance emerges naturally from continuous Hebbian learning in autonomous neural networks. Sci Rep 8:8939

70. Vigetti D, Andrini O, Clerici M, Negrini D, Passi A, Moriondo A (2008) Chondroitin sulfates act as extracellular gating modifiers on voltage-dependent ion channels. Cell Physiol Biochem 22:137-146

71. Villa KL, Berry KP, Subramanian J, Cha JW, Oh WC, Kwon HB, Kubota Y, So PT, Nedivi E (2016) Inhibitory synapses are repeatedly assembled and removed at persistent sites in vivo. Neuron 89:756-769

72. von Holst A, Sirko S, Faissner A (2006) The unique 473HD-Chondroitinsulfate epitope is expressed by radial glia and involved in neural precursor cell proliferation. J Neurosci 26:4082-4094

73. Wang YC, Dzyubenko E, Sanchez-Mendoza EH, Sardari M, Silva de Carvalho T, Doeppner TR, Kaltwasser B, Machado P, Kleinschnitz C, Bassetti CL, Hermann DM (2018) Postacute delivery of GABAA alpha5 antagonist promotes postischemic neurological recovery and peri-infarct brain remodeling. Stroke 49:2495-2503

Publisher's Note Springer Nature remains neutral with regard to jurisdictional claims in published maps and institutional affiliations. 